\title{
FRANK H. KNIGHT LE RISQUE COMME CRITIQUE DE L'ÉCONOMIE POLITIQUE ${ }^{1}$ \\ Pierre-Charles Pradier et David Teira Serrano
}

RÉSUMÉ : Nous proposons dans cet article de rechercher chez les prédécesseurs de Frank Knight un peu de lumière pour éclairer sa distinction entre risque et incertitude. D'une part, la lecture des économistes qui l'ont précédé permet de comprendre sa situation dans la polémique sur la répartition. D'autre part, l'examen des sources philosophiques qui informent sa théorie de la connaissance conduit à analyser l'opposition risque/incertitude comme une opposition théorie de la probabilité/ théorie de la connaissance (imparfaite). Le recours à l'épistémologie de Knight fait saisir en quoi sa démarche philosophique le mène à conclure par l'absurde la polémique sur la répartition, et l'autorise alors à proposer une reconstruction de la théorie économique comme science des choix rationnels.

MoTS-CLÉs : choix rationnel, épistémologie, incertitude, probabilité, répartition, risque.

ABSTRACT: In this paper we try to throw some light on the Knightian distinction between risk and uncertainty attending to a number of previous contributions to philosophy and economics. On the economists' side, we propose to read Knight's distinction in the light of the distribution controversy that took place in the Quarterly Journal of Economics at the end of the XIX ${ }^{\text {th }}$ century. On the philosophers' side, the pair risk/uncertainty can be analysed in view of Knight's epistemological sources, as opposing a sort of probabilistic positivism to an agnostic variety of positivism. His philosophical commitments can be of use in explaining why Frank Knight closes down the distribution controversy, proposing instead a reconstruction of economics as a rational choice science.

KEYWORDS : rational choice, epistemology, uncertainty, probability, distribution, risk.

1. Des versions préliminaires de cet article ont été présentées au séminaire d'histoire des probabilités et des statistiques du Centre d'analyse et de mathématiques sociales (CAMS) et au colloque European conference on the history of economics (ECHE) en 1997. Les auteurs tiennent à remercier les participants de ces événements (en part., Éric Brian, José-Luis Cardoso, Athanase Drivas, Philippe Fontaine, Mary Morgan et Roy Weintraub) pour la bienveillance de leurs remarques. Toutes les erreurs résiduelles sont évidemment à imputer aux seuls auteurs. Enfin, pour plus de précisions sur les références citées dans les notes, se reporter à la liste finale, infra, p. 113-116.

Revue de synthèse : $4^{\mathrm{e}}$ S. $\mathrm{n}^{\text {os }} 1-2$, janv.-juin 2000, p. 79-116. 
Zusammenfassung: In dem vorliegenden Artikel versuchen wir einige Anhaltspunkte zu finden, um die knightianische Unterscheidung zwischen Risiko und Ungewißheit aufgrund der Werke seiner Vorgänger zu beleuchten. Vom Standpunkt der Wirtschaftswissenschaftler aus können wir die Unterscheidung im Licht der Verteilungskontroverse interpretieren, die im Quarterly Journal of Economics am Ende des 19. Jahrhunderts stattfand. Von der philosophischen Seite aus können wir die Unterscheidung zwischen Risiko und Ungewißheit im Hinblick auf Frank Knights epistemologische Quelle analysieren, indem wir wahrscheinlichen Positivismus und agnostischen Positivismus gegenüberstellen. Die Analyse der Epistemologie Knights erlaubt uns zu erklären, warum er die Verteilungsdebatte für absurd hält und statt dessen eine Rekonstruktion der Wirtschaftswissenschaft als eine Wissenschaft der rationalen Wahl vorschlägt.

STICHWÖRTER : rationale Wahl, Epistemologie, Ungewißheit, Wahrscheinlichkeit, Verteilung, Risiko.

RESUMEN : En el presente artículo intentamos obtener algunas claves para iluminar la distinción knightiana entre riesgo $e$ incertidumbre a partir de la obra de sus predecesores. Por parte de los economistas, podemos interpretar la distinción a la luz del debate sobre la distribución que tuvo lugar en el Quarterly Journal of Economics a finales del XIX. Por otra parte, atendiendo a sus fuentes filosóficas, podemos analizar la distinción entre riesgo $e$ incertidumbre en términos de teoría del conocimiento oponiendo positivismo probabilístico a positivismo agnóstico. El análisis de la epistemología de Knight nos permite explicar por qué cierra por absurdo el debate sobre la distribución, proponiendo a cambio una reconstrucción de la teoría económica como ciencia de la elección racional.

PALABRAS CLAVES : elección racional, epistemología, incertidumbre, probabilidad, distribución, riesgo.

Pierre-Charles Pradier, né en 1971, est maître de conférences à l'UFR d'économie de l'université Paris-I.

Adresse : Université Paris-I, 90 rue de Tolbiac, F-75013 Paris.

Courrier électronique : pichalecha@aol.com

David Teira Serrano, né en 1972, doctorant au département de Philosophie de l'UNED à Madrid, prépare une thèse sur quelques idées économiques et philosophiques de l'école de Chicago (Knight, Friedman, etc.).

Adresse : Dpto logica y filosofía de la ciencia, UNED, Senda del Rey s/n, E-28040 Madrid.

Courrier électronique : dteira@ctv.es 
Frank Hyneman Knight est connu de tous les étudiants en économie pour sa distinction entre les concepts de risque et d'incertitude. C'est souvent par cette distinction que les économistes commencent leurs exposés sur le risque, et c'est à peu près le seul point qui fasse l'unanimité dans la profession. Mais cette unanimité n'est qu'une façade, car si tous s'accordent à mettre en exorde la dyade knightienne, chacun lui donne un sens particulier. Quelques articles récents ont ajouté au trouble en notant les « erreurs» de « la tradition» et en proposant des interprétations «nouvelles » : qu'en est-il alors de cette distinction? Ne s'est-elle perpétuée que pour des raisons institutionnelles, en raison de la forte position de Knight, qui après avoir dirigé le département d'économie de l'université de Chicago (qui a formé tant de prix Nobel, de Milton Friedman à Robert Lucas, en passant par George Stigler), a été membre fondateur (et influent) de la très libérale Société du mont Pèlerin et encore présidé en 1950 l'American Economic Association? Ou au contraire, est-ce un effet de la puissance analytique des concepts introduits par Knight dans un débat qui, avant lui, manquait de clarté?

Alors que l'historiographie a tenté d'éclairer le sujet en privilégiant l'interprétation de chaque partie de l'œuvre knightienne en référence à sa totalité (sans prêter attention aux changements de l'environnement historique), nous avons choisi de lire Risk, uncertainty and profit à la lumière de son époque. Pour cela, il convient de s'intéresser d'abord au contexte sociopolitique qui justifie les travaux de Knight, autant qu'à ce que ce contexte a suscité chez ses devanciers : aussi étrange que cela puisse paraître, ce point intéresse rarement les économistes, fussent-ils historiens de leur discipline. Il apparaît alors que la polémique du Quarterly Journal of Economics, roulant sur la justice sociale et son expression théorique (la question de la répartition), constitue un élément clé de ce contexte, qu'il est nécessaire de présenter en premier lieu. Dans la suite de notre étude, nous avons choisi d'analyser la différence spécifique de Knight, économiste formé au département de philosophie de Cornell (dont il a été exclu avec perte et fracas en 1913), mais toujours tenté de revenir à la philosophie, comme en témoigne sa nomination en 1945 à un poste de philosophie ${ }^{2}$, après la publication de papiers dans cette discipline ${ }^{3}$. Il peut sembler que l'originalité de la démarche de Knight provienne de cette particularité; il est donc légitime de rechercher les fondements philosophiques d'une distinction qui est pour Knight caractéristique de la théorie de la connaissance.

2. WICK, 1973, p. 513.

3. KNIGHT, 1935 et 1947. 
Après l'amont, l'aval : la dernière partie de ce travail sera consacrée à un examen des conséquences et de la portée de l'opposition entre risque et incertitude. Ce qui pourrait sembler un détail technique induit non seulement une réponse au problème posé à Knight par ses devanciers (la théorie de la répartition), mais encore une redéfinition de l'objet de la science économique.

KNIGHT, ENFANT DE LA POLÉMIQUE DU QUARTERLY JOURNAL OF ECONOMICS

Avant de présenter la polémique du Quarterly Journal of Economics, qui est le creuset de la réflexion économique de Knight, il convient de s'intéresser brièvement à son contexte historique.

La fin du $\mathrm{XIX}^{\mathrm{e}}$ siècle est marquée par des troubles sociaux récurrents en Amérique du Nord. Claude Julien et Marie-France Toinet en égrènent la litanie :

«C'est alors la grande époque de l'agitation sociale aux États-Unis. La grande grève de 1877, qui atteint tous les centres ferroviaires et voit la ville de Pittsburg occupée par les insurgés; les événements de Haymarket en 1886; les grèves de Homestead en 1892, de Pullman en 1894 et de Cœur d'Alene de 1892 à 1899; la véritable "guerre de trente ans" du Colorado entre 1884 et 1914 et les grèves des métiers du bâtiment en 1909-1910 ne sont que les temps forts qui rythment, année après année, le florilège de l'histoire ouvrière américaine. Ils manifestent la violente opposition entre ouvriers et patrons des grandes industries naissantes ${ }^{4}$.»

Cette situation n'est pas propre aux États-Unis : l'encyclique Rerum Novarum, publiée en 1891 par Léon XIII, témoigne de l'actualité de la question sociale sur le vieux continent. De Fourmies (1891) à Draveil (1904), des grèves endeuillées par l'intervention de «la troupe » écrivent en lettres de sang la jeune histoire de la grève.

Ce serait une erreur de croire qu'un questionnement de nature sociale «émerge » seulement au tournant du siècle. En 1820, David Ricardo proposait déjà une définition de l'économie centrée sur le sujet :

«L'économie politique est selon vous [Malthus] une enquête sur la nature et les causes de la richesse. Je pense qu'il vaudrait mieux l'appeler une enquête au sujet de la division du produit de l'industrie entre les classes qui concourent

4. Julien-Toinet, p. $386 \mathrm{~b}$. 
à sa formation. Aucune loi ne peut être établie concernant la quantité, mais on peut en assigner une suffisamment correcte aux proportions. De jour en jour je suis plus convaincu que la première étude est vaine et illusoire et que la seconde constitue l'objet propre de la science ${ }^{5}$.»

Ricardo insiste ici sur le recadrage qu'il opère par rapport à la problématique de Thomas Malthus, qui fut aussi celle d'Adam Smith (c'est à lui que renvoie l'expression «enquête sur la nature et les causes de la richesse ${ }^{6}{ }$ ) ou de Jean-Baptiste Say : la question de la répartition qui était subalterne devient primordiale. Mais cette insistance ne doit pas dissimuler un accord entre auteurs dits classiques sur la manière de concevoir la répartition. En effet, la question de la répartition du produit concerne les « classes », selon le mot de Ricardo (et bientôt, de Karl Marx), au nombre de trois : les propriétaires, les capitalistes, les travailleurs. Chaque classe offre une contribution particulière au produit, un «facteur » de production propre : la terre, le capital, le travail (respectivement). Chaque facteur reçoit un revenu qui lui est propre (et dont la détermination est spécifique) : la rente, le profit, le salaire.

Même si la vision classique de la répartition ne constitue pas ici le sujet - elle a été étudiée par Jean Marchal et Jacques Lecaillon dans une somme qui fit date ${ }^{7}$ - il faut cependant signaler quelques caractéristiques utiles pour la suite. D'abord, il faut insister sur le caractère global, «macroéconomique » de la problématique des économistes classiques : il s'agit de répartition entre classes sociales participant à la production nationale dans son ensemble, on l'a dit, et non entre agents participant à une production particulière. Il y a donc assez peu d'éléments «microéconomiques » chez les classiques, bien que Smith détaille comment le taux de profit d'un capitaliste (prime de risque) ou le salaire d'un travailleur dépendent du risque. Pour ce dernier, c'est le cas si son activité est marquée soit par un caractère saisonnier (compensation du risque de chômage), soit par une probabilité de succès assez faible (comme par exemple dans la carrière lyrique $)^{8}$. Le risque est un des rares éléments microéconomiques de l'analyse classique, il permet d'expliquer différentiellement une rémunération qui paraît anormalement élevée. Mais de façon générale, les auteurs ne s'intéressent pas à la détermination d'une rémunération en particulier (et de fait les considérations sur le risque de Smith ont disparu chez Ricardo).

5. RicARDo, 1952, p. 278-279.

6. SMITH, 1776.

7. Marchal-Lecaillon, 1958, voir, en part., vol. III. Les volumes 1 et 2 font le point sur les aspects empiriques du phénomène.

8. Voir Pradier, 1998, p. 145 sqq. ; Smith, 1776, liv. I, chap. vi et X, p. 151-152 et 202-214. 
Si donc les questions de nature sociale intéressent l'économie politique depuis les premiers temps de sa formation, quelle est la particularité américaine? Elle tient d'abord à un trait distinctif du capitalisme américain. Les «Robber barons» que furent Daniel Drew, Cornelius Vanderbilt, Jay Gould et les autres figures de proue du « capitalisme sauvage ${ }^{9} »$ n'ont pas autofinancé la construction de leurs empires, ils ont au contraire fait un large appel à l'épargne, généralement par le biais d'émissions obligataires massives. De ce fait, à l'inverse des capitalistes européens qui ont marqué la révolution industrielle, les Américains font essentiellement travailler l'argent des autres, ce sont donc les premiers managers. Si l'on ajoute l'absence d'aristocratie et la distribution de terres aux colons de la frontière, il apparaît que la spécificité américaine implique l'échange d'une classe sociale pour une autre. Le propriétaire terrien des Européens est remplacé chez les auteurs américains par l'entrepreneur ${ }^{10}$; on précisera ultérieurement le facteur de production et le type de revenu qui lui reviennent.

Une autre différence est à mettre au compte de l'Amérique des années 1890 (non plus du côté des faits, mais de la théorie) : les économistes s'y sont volontiers exprimés «en termes néo-classiques ». Cette expression assez vague ${ }^{11}$ indique, entre autres, une rupture fondamentale dans la façon de concevoir l'économie, avec l'adoption d'une approche microéconomique. Ce choix méthodologique conduit, en matière de répartition à s'intéresser non plus à des classes sociales (représentées par des «facteurs »), mais à des individus particuliers, apportant à une production donnée un panier d'intrants (le changement de nom dénote le changement d'approche) : le souci de réalisme transparaît parfois dans des études de cas $^{12}$, au demeurant assez rares. L'approche désagrégée conduit à raffiner la nomenclature des intrants nécessaires, et il n'est plus possible en général de leur faire correspondre une classe sociale. La problématique de la répartition s'oriente donc vers une enquête assez technique ${ }^{13}$, où l'on doit mesurer la contribution de chacun (ou de chaque intrant) au produit total. La comparaison de cette contribution de chacun à la production avec le revenu qu'il en retire est naturelle. La question fondamentale de la répartition, de la justice sociale comme on est sur le point de le dire ${ }^{14}$, est, selon

9. Sur ce sujet, il est impossible de ne pas renvoyer à Debouzy, 1972.

10. À l'évocation de ce substantif, il semble impossible de ne pas citer Vérin, 1982.

11. La pensée néo-classique débute vers 1870-1873 avec les travaux de Stanley Jevons en Angleterre, Carl Menger en Autriche et Léon Walras en France. Pour une présentation simple mais complète, voir GUERRIEN, 1999.

12. Le meilleur exemple, bien qu'il soit un peu tardif, est Marshall, 1923.

13. Il ne s'agit évidemment pas d'enquêtes réelles, puisqu'il est impossible de mesurer dans les faits la contribution de chacun (comme l'a montré KNIGHT, voir infra, p. 102-103). Les économistes se donnent des entreprises fictives représentées par des fonctions mathématiques. Voir Guerrien, 1999 pour des exemples.

14. Cette expression n'est toutefois pas attestée avant 1919. 
ces auteurs, de savoir si la contribution à la production et la rémunération tirée de cette production sont égales.

Mais de quels auteurs est-il question? Entre 1891 et 1904, plusieurs dizaines d'articles constituent ce qu'on pourrait appeler la polémique du Quarterly Journal of Economics. Cet épisode met en scène des figures de proue de la théorie économique néoclassique, comme Eugen von BöhmBawerk (en Autriche) ou John Bates Clark (figure dominant la scène américaine), mais aussi des intervenants de stature plus modeste : Thomas Carver, Frederick B. Hawley ou John Haynes, qui contribuent cependant à un aspect important de la question. Jürg Niehans a produit sur le sujet un article exploratoire $^{15}$; il décrit une série de «combats singuliers » engagés par Böhm-Bawerk au terme de laquelle s'achève la constitution d'une «théorie de l'intérêt» qui peut «être considérée à bien des égards aujourd'hui encore comme "définitive" " ${ }^{16}$. L'emploi de cette dernière épithète révèle les préoccupations de Niehans, qui le conduisent à négliger le rôle d'un illustre inconnu (qui ne possède pas même son entrée dans le Palgrave, le dictionnaire économique de référence), Hawley. Ce dernier a mis le feu aux poudres à l'été $1891^{17}$, en critiquant l'ouvrage de Böhm-Bawerk. La polémique qu'il ouvre a pour objet de savoir si le profit rémunère le seul capital ou s'il faut ajouter un facteur supplémentaire qui correspondrait au travail des entrepreneurs.

Il faut signaler que l'effet de la situation politique et sociale de l'époque, si elle est mentionnée à l'occasion par les commentateurs ${ }^{18}$, est évidente pour les acteurs. Chez certains, comme Frank Taussig, l'urgence de la question sociale ne transparaît que par allusions :

«La force que les syndicats donnent aux travailleurs dans les négociations avec les employeurs n'a jamais été remise en cause même au plus fort de la confiance dans les lois naturelles qui gouvernaient en principe les phénomènes économiques en général et les salaires en particulier. Personne ne songerait à en douter à cette époque moins conservatrice ${ }^{19}$. »

L'époque serait donc «moins conservatrice » parce qu'elle pose la question sociale au point de remettre en cause les « lois naturelles », c'est-à-dire les enseignements de l'économie politique. Le très conservateur Taussig

\footnotetext{
15. NieHANS, 1991.

16. Niehans, 1991, p. 567 et 569 .

17. Hawley, 1891. Signalons que Niehans, 1991, ne mentionne pas Hawley et fait débuter la polémique en 1892.

18. Sur Clark, voir Morgan, 1994, plus intéressée à la négociation entre les parties qu'à la théorie de la répartition à proprement parler. Ross, 1991, rapporte également les débats à l'origine de la constitution de l'American Economic Association. Aucun de ces auteurs n'inscrit cependant Knight dans le fil de la polémique du Quarterly Journal of Economics.

19. TAussig, 1895 , p. 93.
} 
est d'ailleurs prudent dans ses formulations, comme s'il rapportait au style indirect les arguments des différentes parties. Mais ce détachement apparent fait exception parmi les économistes américains, qui affirment clairement l'enjeu de leurs travaux. Ainsi Clark déclare-t-il dès les premières pages de sa Distribution of wealth, qui constitue l'évangile de la pensée néoclassique américaine :

« [...] s'il apparaissait qu'elles [les classes laborieuses] produisent une grande quantité et n'en reçoivent qu'une petite part, elles deviendraient en grande partie révolutionnaires, et elles auraient entièrement le droit de l'être. Le chef d'accusation qui pèse sur la société est celui "d'exploiter le travail" [...] Si cette accusation s'avérait vraie, alors tout homme honnête devrait devenir socialiste $[\ldots]^{20}$.»

Clark explique donc que la vérité de l'économie politique (et de sa critique) se joue sur le terrain de la répartition, et sur ce terrain seul. On ne peut exprimer plus clairement l'importance du problème. Alors que la polémique continue et s'étend, Clark est à son tour l'objet de critiques :

«Le disciple d'Henry George ${ }^{21}$ pourrait bien admettre que la terre crée une part définie du produit, et nier simultanément que le propriétaire terrien ait quelque droit sur elle. Il pourrait aussi admettre que la terre doive être rémunérée sur la base de sa productivité et nier le droit au propriétaire privé de toucher la rente. Ainsi proposerait-il que ce soit l'État qui reçoive la part de la terre. Le socialiste aurait la même position sur tous les instruments de production. Le droit à l'existence de l'ordre social actuel ne dépend donc pas des lois qui gouvernent la répartition fonctionnelle, mais personnelle ${ }^{22}$. »

Cette longue citation de Carver est particulièrement éclairante. Elle réaffirme, s'il était besoin, l'importance du problème de la répartition qui conditionne «le droit à l'existence de l'ordre social». Elle expose les forces en présence : tenants de l'économie politique, populistes favorables à la nationalisation des terres, et socialistes qui proposent la collectivisation de tout l'appareil productif. Enfin, elle indique une direction à suivre pour penser le problème : celle d'une théorie de l'imputation aux intrants (qui permet ensuite de calculer des revenus personnels comme somme des revenus des intrants investis par une personne) et non plus une théorie (classique) de la répartition entre les classes sociales.

La proposition de Carver résume l'aspect de la polémique du Quarterly Journal of Economics sur lequel nous désirons insister. Puisque nous avons

20. CLARK, 1899 , p. 4.

21. Henry George (1839-1897) était un publiciste américain, on aura compris qu'il proposait la nationalisation des terres.

22. Carver, 1901, p. 579. 
fait le point sur le contexte (à la fois social et théorique) de cette controverse, il convient maintenant d'en présenter les développements. Notre attention se portera naturellement sur les contributions décisives dans la formation de la pensée knightienne et de la théorie néoclassique. On a donc retenu deux éclairages sur le sujet : Hawley introduit d'abord l'«assomption du risque » comme un facteur de production supplémentaire (pour rendre compte du rôle des entrepreneurs). Clark l'élimine sous prétexte de statique, et s'appuie sur la notion de rémunération des facteurs à la productivité marginale. Cela l'engage dans la voie de la désagrégation néoclassique; la polémique fait rage, dans l'attente d'un homme providentiel Frank Hyneman Knight.

Il faut toutefois s'arrêter d'abord sur Hawley. Celui-ci ouvre donc les hostilités en exposant, contre Böhm-Bawerk, un point de vue « entrepreneurial ». Celui-ci tient en deux phrases :

«Mais si la science doit expliciter la conception vulgaire du profit, en ce qu'il se distingue des autres types de revenu, elle doit montrer ce que l'entrepreneur fait en vue d'un revenu et qui n'est rémunéré ni par les salaires, ni par l'intérêt, ni par la rente - ce que le professeur Böhm-Bawerk passe complètement sous silence. Justement, cette fonction industrielle particulière de l'entrepreneur se trouve en ce qu'il est la personne qui délivre les autres du risque ${ }^{23}$. »

Hawley distingue ainsi un «nouveau » facteur de production (celui-là même qui avait déjà été identifié par Turgot), caractéristique d'une classe sociale (les entrepreneurs) et sanctionné par un revenu particulier (le profit). Mais, dès l'année suivante, il doit affronter la critique de Clark.

Clark démontre en deux points que le «nouveau » facteur de production n'existe pas. Le premier temps de la démonstration se veut juridique :

«Il va sans dire que le danger des affaires retombe sur le capitaliste. L'entrepreneur, comme tel, a les mains vides. Personne ne peut prendre un risque s'il n'a rien à perdre. Si l'affaire court à sa perte, c'est l'offreur du capital qui en souffre $[\ldots]^{24}$.»

Clark, à l'opposé des auteurs européens ${ }^{25}$, retire donc la responsabilité du capital des mains de l'entrepreneur. Ce changement de perspective tient probablement à la diffusion de la responsabilité limitée aux États-Unis (au contraire, les entrepreneurs européens autofinancent depuis le XVIII siècle

23. CARver, 1901, p. 283

24. Clark, 1892, p. 46.

25. C'était le cas d'Adam Smith et de Jean-Baptiste Say, en particulier. Ce dernier est souvent considéré comme un précurseur de Knight, voir Fontaine, 1999, ou Pradier, 1998, p. $150-154$. 
le développement de leurs affaires), avec pour corollaire l'impunité des Robber barons et leur mépris pour les bailleurs de fonds. Puisque le capitaliste prend donc seul les risques, il mérite rémunération. Reste cependant à savoir comment on évalue ce revenu, c'est le second point. Clark pose alors que «les risques des affaires sont rémunérés à leur valeur actuarielle subjective ${ }^{26} \gg$. L'auteur utilise donc la décroissance de l'utilité marginale de la richesse pour calculer les dispositions à payer. Faisant usage du principe de Daniel Bernoulli ${ }^{27}$, il peut comme ce dernier insister sur les avantages de la diversification et du partage de risques. Cependant, Clark est plus prudent que Bernoulli, puisqu'il ne précise pas de fonction d'utilité pour la richesse, ni le statut qu'il lui donne. Il indique donc la méthode pour mesurer, sans offrir la possibilité de l'appliquer (dans un modèle microéconomique).

L'escarmouche entre deux auteurs tourne alors à la bataille rangée. Car après une réponse de Hawley ${ }^{28}$, chacun y va de sa contribution, et le débat perd en clarté. Mieux vaut donc préciser la position définitive de Hawley d'une part, et ce qui en est à peu près accepté par les économistes américains d'autre part.

En ce qui concerne Hawley, un dernier article ${ }^{29}$ tente de mettre un point final à la discussion. Le raisonnement s'articule autour de deux sujets essentiels. D'abord, l'auteur réfute la vision de Haynes ${ }^{30}$ d'une rémunération de l'entrepreneur liée à sa position de marché particulière (et non à sa contribution à la production) :

«Son entrepreneur [celui de Haynes] n'est plus une "variété particulière de travailleur", mais il est devenu une variété particulière de monopoliste, dont les gains proviennent "du fait qu'il est simultanément travailleur et capitaliste, sur la base d'un avantage particulier qu'il a en employant son talent et son capital". Maintenant, comme je l'ai montré ailleurs, les gains de monopole (sauf, peut-être, dans le cas de la rente) ne sont pas une forme distincte de revenu qui puisse être mise sur le même plan que les salaires, l'intérêt, et le profit. En effet, le profit n'est qu'une force distributive et non productive ou créatrice comme c'est le cas de la terre, du travail, et du capital ou de l'entreprise, que l'on considère le profit comme la rémunération de l'entreprise ou de quoi que ce soit d'autre ${ }^{31}$.»

Cette longue citation de Hawley présente un relief particulier. D'abord, Hawley y présente la thèse de la rareté de l'entreprise qui donne un carac-

26. Clark, 1892 , p. 42.

27. Bernoulli, 1731, est une référence pour Marshall et Clark.

28. HaWley, 1893.

29. HaWLEY, 1900.

30. Haynes, 1895, n'est pas un auteur particulièrement remarquable, mais sa remarque témoigne d'une critique souvent faite à Hawley.

31. Hawley, 1893, p. 83. 
tère de monopoliste aux entrepreneurs. On peut évidemment réinterpréter toute la littérature économique depuis Cantillon (et en particulier les contributions de Jean-Baptiste Say et de John Stuart Mill) sous cette lumière. Mais Hawley repousse cette thèse qui ne permet pas de répondre de manière satisfaisante à la question sociale. Par « réponse satisfaisante », les auteurs de l'époque entendent une réponse qui montre l'adéquation entre production et répartition. Montrer que la situation de monopole permet de s'attribuer un surplus aux dépens des autres participants à la production ne permet pas de résoudre la question de manière satisfaisante. On doit donc réfléchir dans un cadre de concurrence parfaite (sans monopole d'aucune sorte), en posant que l'entreprise est au contraire un facteur abondant: nombreux sont les travailleurs capables d' « entreprise ${ }^{32} »$.

Le second point important que Hawley réaffirme est le caractère fondamental de la distribution fonctionnelle (c'est-à-dire la répartition entre les facteurs, et non les individus). Comme le problème du monopole, ce point avait été présenté par l'auteur dès 1891 , mais il est réaffirmé sans cesse de façon allusive. L'exposé le plus clair tient en quelques lignes :

«Il n'est probablement pas de principe plus impératif pour la science économique que la règle selon laquelle les classifications et les distinctions de la science, pour être fructueuses, doivent se fonder sur la fonction. Dès que nous cherchons à sélectionner des individus concrets ou des phénomènes concrets, nous sommes perdus. On ne trouvera pas un individu qui ne soit engagé dans la production de plusieurs manières. Même le travailleur démuni de propriété compte un élément de risque dans son environnement : il ne peut jamais être sûr d'être toujours employé ${ }^{33} »$.

Hawley réaffirme donc l'importance de la vision fonctionnelle de la répartition caractéristique de l'approche classique. La problématique de l'imputation technique à des intrants sans contrepartie sociologique est pour lui la perte de l'économie, en ce qu'elle ne permet évidemment pas de répondre à la question sociale. Aussi s'avoue-t-il « déçu » que Haynes, ayant constaté qu'il serait logique de développer entièrement l'idée de « répartition selon les fonctions exercées ${ }^{34}$, ne mène à bien cet exercice. En effet, Haynes doute qu'un tel «système » puisse être «d'une quelconque valeur pour la science ${ }^{35}$. Hawley tient à sa position, mais doit concéder ce qui constitue un changement manifeste depuis Ricardo : le modèle théorique est entière-

32. Si l'entreprise individuelle n'est pas plus développée (si, réciproquement, les salariés sont si nombreux), c'est à cause d'une difficulté dans l'accès au capital (au crédit). Ce problème trouve évidemment une solution à long terme chez les auteurs libéraux comme Say et Mill, voir Pradier, 1998, p. 149 sqq.

33. Hawley, 1891, p. 290.

34. HaWley, 1900, p. 78.

35. Haynes, 1895, p. 423. 
ment distinct de la réalité, et ce n'est plus qu' «aidé par la statistique ${ }^{36}$ » que l'on peut déterminer l'imputation réelle du produit. Cet échec de l'auteur conditionne bien évidemment la réception de ses thèses.

En effet, la communauté des économistes américains, si elle s'accorde à soutenir la première thèse - il faut raisonner en concurrence parfaite même si une interprétation de la réalité en terme de monopole semble aussi plausible - ne peut évidemment plus soutenir la seconde. L'avis de Haynes est largement partagé : à quoi servirait un modèle théorique qui n'apprenne rien sur la réalité? Aussi l'héritage paradoxal de Hawley, comme son repli sur la «statistique » le laissait entrevoir, est-il d'avoir fait admettre la multiplicité des facteurs de production, d'avoir contribué à la vision désagrégée des néoclassiques. Lui-même insistait sur le seul risque, mais dans son article de 1900, il doit faire des concessions, en distinguant risque entrepreneurial, ce qu'il appelle entreprise, risque assuré et risque du spéculateur. Le premier a convaincu les économistes à la suite de l'article de Clark déjà cité ${ }^{37}$, les seconds gagnent leur faveur après une discussion à l'American Economic Association ${ }^{38}$. Hawley concède ces distinctions pour maintenir sa définition de l'entrepreneur ${ }^{39}$, mais ceci n'a pas grand intérêt. Le fait est que la tripartition classique des facteurs est en train d'éclater. Car, à côté des trois modalités du risque, on trouve également la coördination, présentée par Clark et Haynes. La multiplication des intrants fait alors voler en éclats l'ancienne théorie de la répartition, et la construction de la théorie néoclassique de l'imputation est en marche. La seconde pierre de cet édifice est due à Clark.

Il peut sembler curieux de consacrer un temps à la présentation de la pensée de Clark, dont on vient d'expliquer qu'il a expulsé le risque de la théorie de la répartition. Il semble pourtant que Clark ait eu une influence directe et très importante sur la synthèse knightienne (voir infra, p. 92-93). Cela dit, il apparaît que Clark s'oppose fondamentalement à Hawley, de par la conception même du rôle de l'entrepreneur et du capitaliste qu'il défend. En effet, il cantonne l'entrepreneur dans le rôle d'un coordinateur. Il convient toutefois d'insister sur le caractère apparent et non fondamental du désaccord entre Clark et Hawley.

La « découverte ${ }^{40} »$ de Clark, celle qui fonde son édifice théorique, est une astuce mathématique incomplète qui lui permet de résoudre le problème de la répartition. Clark $a$, en effet, le sentiment que si l'on paye le

36. Hawley, 1900, p. 78.

37. Clark, 1892.

38. Voir EMERY, 1898. Le compte rendu de cette communication rapporte aussi les propos de Arthur J. Hadley, Thomas N. Carver, John Rogers Commons et Franklin Giddings.

39. Voir, p. ex., Hawley, 1900, p. 100.

40. Clark découvre en fait la rémunération à la productivité marginale quelque temps après WICKSTEED, 1894; voir infra, p. 91. 
travail à sa productivité marginale et que l'on donne le surplus au capitaliste, ou à l'inverse, si l'on paye le capital à sa productivité marginale en laissant le surplus au travail, ces deux opérations sont identiques. Dans les deux cas, les plus-values sont égales. Puisque le rôle des facteurs est symétrique, on ne peut parler d'exploitation d'un facteur par l'autre. Il apparaît alors que rémunération et contribution à la production des deux facteurs sont égales. Clark croit ainsi avoir résolu la question sociale en montrant la justice de la répartition. Malheureusement, il est ignorant d'une condition nécessaire (et suffisante) pour que son théorème soit vrai : il faut supposer l'homogénéité de degré un des fonctions de production (la condition d'Euler). Mais ce genre de raffinement est étranger à la méthode de l'auteur qui procède par une étude graphique, en terme d'aires sous des courbes.

Du fait de la méthode graphique qu'il emploie (avec des courbes dans le plan), Clark doit se limiter à étudier la répartition entre deux facteurs. Pour ce faire, il range la terre dans le capital. Mais il doit aussi éliminer les autres facteurs de production, ceux-là mêmes que l'analyse de Hawley commence à distinguer. Le meilleur moyen est alors de réserver le risque aux capitalistes - ce qui permet de faire apparaître une gamme de taux d'intérêt que distinguent les primes de risque — et la fameuse coördination aux entrepreneurs. Clark observe alors que dans un environnement dynamique, l'action de l'entrepreneur est nécessaire, mais dans un contexte statique où la répétition entraîne la connaissance parfaite des prix et des quantités, la coördination n'est plus nécessaire. Du coup, en statique, il ne reste plus que deux facteurs distincts : la méthode de Clark peut alors s'appliquer.

L'opposition de Clark à Hawley semble donc entièrement déterminée par l'urgence où celui-ci est de résoudre le problème de la répartition, ce qui le conduit à des expédients théoriques. Il est probable, que si on lui offrait le moyen de déterminer conjointement la rémunération de plusieurs facteurs, il se montrerait plus souple sur le comptage des facteurs. De fait, la solution existe, bien que Clark n'en ait pas encore conscience.

Après quinze ans de controverse, Francis Y. Edgeworth prend la plume pour écrire un très long article dans le Quarterly Journal of Economics ${ }^{41}$, article qui met un terme provisoire à la polémique. Sa culture économique et mathématique lui permet d'offrir aux Américains une synthèse qui leur était inaccessible. D'abord, Edgeworth présente de manière complète et définitive la méthode de la productivité marginale, qu'il attribue à Philip Wicksteed $^{42}$. L'usage des dérivées partielles en lieu et place des études

41. EDGEWORTH, 1904.

42. WiCKSTEED, 1894. 
graphiques permet d'étendre l'analyse à un nombre quelconque de facteurs. Mais Edgeworth met également l'accent sur la nécessité de l'hypothèse mathématique cruciale (l'homogénéité de degré un, qui suppose des rendements d'échelle constants). Suite à ce constat, Edgeworth s'amuse de la naïveté de Clark :

«Cette magnifique généralisation rappelle la jeunesse de la philosophie. La justice est un cube parfait, disait le sage antique; et la conduite rationnelle est dictée par les fonctions homogènes, ajoute le savant moderne. Une théorie qui conduit à des conclusions aussi paradoxales doit sûrement être énoncée avec précaution ${ }^{43}$.»

L'argument d'Edgeworth ne fait qu'insister sur l'impossibilité d'introduire l'entrepreneur dans le modèle de (Wicksteed-)Clark. Cela conduit à penser que la théorie de Clark est encore plus éloignée de la réalité que l'auteur ne le soupçonnait. D'une certaine façon, peu importe : la question sociale est résolue dans un monde imaginaire.

La polémique du Quarterly Journal of Economics s'achève donc piteusement, et les néoclassiques américains apparaissent finalement dans une position de faiblesse relative. Clark vieillissant, la vogue marginaliste passe momentanément aux États-Unis malgré l'énergie déployée par Irving Fisher. Comme le montrent Bruna Ingrao et Giorgio Israel, la mode passe à l'étude du cycle des affaires ${ }^{44}$, activité plus descriptive et moins théorique. L'Europe est en proie à la Grande Guerre quand l'Amérique découvre les voies d'une économie statistique et institutionnaliste.

Il se trouve pourtant un étudiant d'Alvin Johnson - lui-même autrefois thésard et secrétaire de Clark - volontaire pour un nouvel essai sur ce sujet en apparence passé de mode. La thèse qu'il achève en 1916, et modifie sérieusement pour la publier en 1921, brille aujourd'hui au firmament des classiques de l'analyse économique. On doit cependant concéder, suivant l'oxymore cornélien, que la clarté knightienne est assez obscure ${ }^{45}$, au moins dans ses nouveautés; c'est plutôt comme synthèse des travaux précédents que l'œuvre de Knight parait lumineuse. Ainsi, malgré la critique impitoyable de Clark et de Hawley qu'il assène aux premières pages de Risk, uncertainty and profit ${ }^{46}$, Knight reprend l'essentiel des acquis de ses devanciers. La distinction entre statique et dynamique (reprise de Clark), comme la distinction entre risque et incertitude (implicite chez Hawley) étaient présentes dans la polémique du Quarterly Journal of Economics.

\footnotetext{
43. EDGEWORTh, 1904, p. 31.

44. Ingrao et IsRaEL, 1987 , p. 245-246.

45. Faut-il rappeler CorneILle, Le Cid, acte IV, scène III, Rodrigue : "Cette obscure clarté qui tombe des étoiles »?

46. KNIGHT, 1921, p. 31-48.
} 
La distinction statique/dynamique chez Clark a déjà été rapidement présentée ici, il convient cependant de la préciser pour introduire l'apport de Knight. Chez Clark, statique et dynamique sont distinguées (entre autres) par la nature des risques spécifiques : il existe des risques statiques et dynamiques. Le premier cas est illustré par l'exemple du risque d'incendie, le second par l'apparition de nouveaux «processus » de production, ou de nouveaux biens ${ }^{47}$. Pour Clark, les distributions de probabilité liées à ces événements sont connues, mais la nouveauté introduit tout de même le besoin pour une mystérieuse coördination dont on se demande vraiment en quoi elle consiste (sinon qu'elle justifie le revenu de l'entrepreneur). L'examen de la structure des ouvrages de Clark et de Knight montre qu'elle est identique. Dans le cas statique, la connaissance parfaite (fût-elle probabiliste) conduit à l'élimination du profit; il n'y a donc pas de place pour l'entrepreneur. Dans le cas dynamique, l'existence de changements dans l'économie aboutit à la réalisation d'un profit qui rémunère la coördination (chez Clark) ou l'entreprise (chez Knight) en tant que facteurs, c'est-à-dire les entrepreneurs en tant que personnes. Les noms sont différents, mais l'idée est la même.

Knight ne semble cependant pas très satisfait de la distinction entre statique et dynamique chez Clark, au moins d'un point de vue épistémique. Dans tous les cas, les agents économiques connaissent les distributions de probabilités, ils devraient donc se comporter de la même façon. Avant de suggérer une distinction épistémique plus tranchée (entre une statique où les probabilités sont connues et une dynamique où elles doivent être devinées), Knight cible, dès le début de son ouvrage ${ }^{48}$, ce point faible de son prédécesseur. À quels arguments recourt-il? À ceux qui étaient ceux de Hawley dans la polémique du Quarterly Journal of Economics. Du coup, on serait tenté de croire que la théorie de Knight consiste à utiliser le point de vue de Hawley pour faire fonctionner la distinction statique/dynamique de Clark.

Mais Knight profite des reproches à Clark pour assimiler ensuite l'opinion de Hawley à celle de son adversaire : «[...] les deux parties de la controverse [du Quarterly Journal of Economics] sont tombées dans la même confusion, en faisant l'hypothèse que la "valeur actuarielle" des risques était connue des entrepreneurs ${ }^{49}$. $\gg$ Ce reproche biaisé est pris pour argent comptant par les économistes ${ }^{50}$. Si la «valeur actuarielle subjective » de Clark est ici directement visée, la dernière intervention de Hawley dans le Quarterly Journal of Economics semble donner tort à

47. Clark, 1892, p. 52-53.

48. KNIGHT, 1921, p. 37.

49. KNight, 1921, p. 43.

50. Leroy et Singell, 1987, p. 396. 
Knight. Hawley y prend l'exemple des spéculations sur le coton : il y décrit d'abord les risques de cette spéculation comme n'étant pas assurables ${ }^{51}$, mais remarque ensuite la possibilité de couvrir par des achats et des ventes à terme les opérations au comptant. Par la suite, il montre que la spéculation étant un jeu à somme nulle, certains réussissent où d'autres se ruinent, mais en tout cas, à un moment donné, il existe des avis divergents sur l'avenir, des probabilités subjectives différentes si l'on veut.

Il semble donc que Knight ait volontairement confondu les positions de Clark et de Hawley pour mettre en valeur sa distinction entre statique et dynamique, caractérisée par une distinction des risques que nous avons appelés avec Clark, statiques et dynamiques. Knight n'est bien sûr ni le seul, ni le dernier économiste à polémiquer ainsi, et dans le même registre, John Maynard Keynes a souvent fait de même en prêtant à ceux qu'il appelle «classiques» des idées parfois caricaturales. Si la distinction knightienne n'apparaît pas comme telle chez Hawley, elle est implicite, comme le montre l'exemple des marchés financiers. Il faut cependant concéder que Knight ne reprend pas seulement le point de vue de Hawley, il le clarifie en rendant explicite une distinction épistémique qui n'était qu'implicite.

Knight poursuit dans la voie ouverte par Hawley : un investissement permet d'obtenir un profit, mais pas de façon certaine, le profit est donc la récompense (incertaine) de la prise de risque.

«Le prix des services productifs constitue le coût de production; mais les changements de l'environnement donnent naissance au profit dans la mesure où ils prennent à revers les anticipations et entraînent des divergences entre ces coûts et des prix de vente, que la concurrence rendrait égaux [sans ces changements $]^{52}$.»

En régime de concurrence parfaite (sans incertitude), tel qu'il est analysé dans la première partie de l'ouvrage de Knight, les agents économiques anticipent parfaitement les possibilités d'arbitrage. Il n'existe donc pas d'espace pour le profit puisque les prix d'achat et de vente sont toujours égaux. On peut considérer cependant que notre connaissance du monde réel est toujours incomplète, et cela seulement permet d'expliquer l'apparition d'un profit. En effet, pour Knight, la possibilité de l'estimation statistique permet à l'entrepreneur de transformer les pertes en coûts fixes grâce à la loi des grands nombres ( «[...] sur la base d'un grand nombre d'entreprises », écrit Knight ${ }^{53}$ ). Par ce biais, on élimine autant les risques que les bénéfices potentiels.

51. Hawley, 1900, p. 97.

52. KNIGHT, 1921, p. 198.

53. KNIGHT, 1921, p. 199. 
Mais les profits existent dans la réalité, et si l'économiste veut en rendre compte, il doit donc postuler un risque mathématiquement irréductible par la loi des grand nombres (et ainsi impossible à prévoir et à provisionner pour l'entrepreneur). Cela revient à dire que les profits découlent des imperfections de la connaissance. C'est ce que Knight tente de faire valoir en créant le concept d'incertitude; il entre ainsi de plain-pied dans la théorie de la connaissance :

«Si l'on veut comprendre le fonctionnement du système économique, on doit examiner à la fois le sens et la portée du concept d'incertitude; à cette fin, c'est une véritable enquête sur la nature et l'usage de la connaissance elle-même qui est nécessaire ${ }^{54}$.»

On perçoit ici la spécificité de Knight par rapport à ses collègues (et devanciers) : si sa démarche n'est pas à proprement parler originale d'un point de vue économique, c'est le fondement philosophique de la distinction risque/incertitude qui est nouveau ${ }^{55}$. Malheureusement, il apparaît rapidement que Knight est tout sauf un homme de système; de plus, il est peu explicite sur ses sources. De ce fait, les lectures de son œuvre sont nombreuses et contradictoires ${ }^{56}$.

Une lecture duale de Knight va être proposée ici. D'une part, la problématique de la répartition, telle qu'elle vient d'être présentée, trouve sa solution (inattendue, il est vrai) grâce à la distinction risque/incertitude. D'autre part, cette même distinction peut être relue à la lumière d'une autre controverse également fin de siècle : le débat sur l'agnosticisme lancé par Emil Du Bois-Reymond dans sa conférence de 1872 « Über die Grenzen des Naturerkennens ». C'est précisément ce sujet qui sera traité en premier lieu, car il est la condition des développements ultérieurs.

LES FONDEMENTS PHILOSOPHIQUES DE LA PENSÉE DE KNIGHT

Autant commencer par dire que nous avons parfaitement conscience du caractère épineux, sinon insoluble, de l'interprétation de la distinction knightienne ${ }^{57}$. Nous ne voulons que suggérer des pistes qui nous ont sem-

54. KNIGHT, 1921, p. 199.

55. Voir cependant FonTAINE, 1999, p. 8.

56. Pour un commentaire d'économiste, voir la discussion de Langlois et Cosgel, 1993, et Leroy et Singell, 1987, in Pradier, 1998; Teira Serrano, 1997, fait le point sur les commentaires philosophiques de McKinney, 1977, ainsi que Emmett, 1992, et Hammond, 1991.

57. De ce fait, nous ne pouvons que saluer les reconstructions, fussent-elles divergentes, comme Емметт, 1992, p. 31. 
blé mener assez loin dans la compréhension de l'auteur, et qui sont suggérées par Knight lui-même dans le chapitre vII de Risk, uncertainty and profit. Dans ce chapitre, que les économistes goûtent peu en raison de sa forte teneur en philosophie de la connaissance, Knight introduit sa distinction entre risque et incertitude à l'aide d'une théorie de la probabilité et d'une théorie de la connaissance. La première permet de comprendre ce que l'auteur entend par risque, quand la seconde développe sa conception de l'incertitude.

Nous savons, tout d'abord, en ce qui concerne la statistique, que Knight fait usage d'un certain nombre de sources (qu'il inventorie dans les notes des pages 212, 221, 222, 224 de son ouvrage). Il nous semble cependant que la Grammar of science de Karl Pearson jouit d'une importance particulière. Knight ne renvoie-t-il pas à ses chapitres Iv et v qui «peuvent être consultés pour justifier le présent chapitre ${ }^{58} »$ ? Ce « présent chapitre » (le septième de Risk, uncertainty and profit) est précisément nommé «les significations de risque et incertitude ». Il convient donc de présenter brièvement l'épistémologie de Pearson avant de discuter sa relation avec celle de Knight.

Pearson est certes positiviste dans la mesure où il considère que l'universalité de la science est fondée sur l'expérience sensorielle, mais d'une variété un peu particulière. Pour lui, le contenu empirique de la science est essentiellement de nature statistique, comme le prouvent ses recherches en biologie. C'est bien cette forme qui garantit l'unité de la science, unité qui consiste « dans sa seule méthode, non pas dans son objet ${ }^{59}{ }$. D' après Pearson, « la classification des faits, la reconnaissance de leur enchaînement et de leur importance relative $»$ serait la «fonction de la science ${ }^{60}$. Pour l'auteur, le concept statistique de corrélation permet de justifier son point de vue, et d'asseoir la validité de la science tout entière ${ }^{61}$. Il suffit pour cela d'admettre que les relations causales (qui sont le critérium de la scientificité) ne sont qu'une modalité des associations perceptives. Or, nous sommes capables de corréler intuitivement des perceptions sensorielles afin de distinguer des niveaux de proximité entre phénomènes, des automatismes perceptifs : "Pour des êtres rationnels qui mènent leur vie dans le temps et l'espace, il est essentiel de discerner des automatismes perceptifs : sans cela la prévision, et donc la conduite rationnelle, sont impossibles ${ }^{62}$.

La causalité d'une part, le hasard d'autre part, ne sont que les limites conceptuelles (et mathématiques) de la corrélation. Afin de corréler scienti-

\footnotetext{
58. KNIGHT, 1921, p. 212.

59. Pearson, 1911, p. 12.

60. Pearson, 1911, p. 6.

61. Pearson, 1911, p. 158 sqq.

62. Pearson, 1911, p. 153.
} 
fiquement deux classes différentes de phénomènes, on groupe leurs manifestations dans les cellules d'une «table de contingence », à laquelle « la meilleure description scientifique des relations entre deux choses peut toujours être rapportée ${ }^{63}$. À l'aide de cette représentation, on obtient une mesure de l'association entre les phénomènes, qui est un point de départ pour des calculs plus complexes. On peut ainsi construire une « classification » des faits qui permette enfin de s'affranchir de toute énigme résistant à la science...

Cela posé, l'influence de Pearson transparaît clairement dans la conception knightienne des statistiques. Pour les deux auteurs, l'estimation statistique est fondée sur la classification : si elle peut être parfaitement homogène, alors elle rejoint la «probabilité a priori», en revanche si l'échantillon est moins homogène, on obtient la «probabilité statistique $»{ }^{64}$. Si l'on suit Knight, l'entrepreneur — agissant comme chacun d'entre nous — va estimer les probabilités des différents événements relatifs à son investissement sans pouvoir classer les cas, car ceux-ci sont complètement hétérogènes :

«Le fait essentiel et caractéristique ici est que chacun des "cas" est si complètement unique qu'il n'en est pas d'autres (du moins pas assez d'autres) pour que l'on puisse construire une classification. Il n'est donc pas possible d'inférer quoi que ce soit de la valeur d'une probabilité réelle face aux phénomènes qui nous intéressent ${ }^{65}$. »

Comme Knight le remarque, même si une estimation numérique probabiliste est disponible, la décision de l'entrepreneur ne peut être analysée en termes statistiques, dans la mesure où les phénomènes — non seulement les phénomènes objectifs que le décideur cherche à appréhender mais aussi les phénomènes mentaux qu'il met en jeu - ne peuvent être classifiés.

On pourrait alors penser que la distinction risque/incertitude recouvre, en termes contemporains, une distinction probabilité objective/probabilité subjective (c'est d'ailleurs l'interprétation classique de Knight chez les économistes). S'il est vrai que le risque, avec les probabilités a priori et statistiques, peut être réduit à la probabilité subjective, il est un peu anachronique et insatisfaisant d'assimiler incertitude et probabilité subjective. Anachronique car le calcul des probabilités subjectives tel qu'il a

63. Pearson, 1911, p. 153. Sur les fondements mathématiques de la méthode de Pearson, voir Armatte, 1995, p. 518 sqq.

64. KNIGHT, 1921, p. 224, écrit : «Probabilité a priori. Elle repose sur une classification absolument homogène de cas en tous points identiques, sauf pour ce qui est des facteurs réellement indéterminés. » À la p. 225, sous le titre «probabilité statistique », Knight écrit : «Le principal trait distinctif, par rapport à la probabilité a priori est qu'elle repose sur une classification empirique des cas. »

65. KNIGHT, 1921, p. 226. 
été développé par Frank Ramsey, Bruno de Finetti et Leonard Savage, n'existe pas encore. Insatisfaisant car dans l'esprit de Knight (comme de Pearson, au reste), cette absence de probabilité objective est le signe d'un manque irrémédiable. D'autant que l'auteur insiste sur le caractère irréductible de notre ignorance : «Le fond de logique et de la psychologie de ces délibérations [probabilistes] est obscur. Voilà un aspect du mystère de la vie et de l'esprit, en tant qu'il est insondable pour la science elle-même ${ }^{66}$. » En incertitude, on ne connaît finalement les estimations que par leur résultat et de ce fait, on ne peut ni les comparer, ni les classifier. Les entrepreneurs eux-mêmes ignorent d'ailleurs comment ils y parviennent ${ }^{67}$.

Est-ce à dire que nous sommes condamnés à une éternelle ignorance? Pas pour Pearson, pour qui l'avancée des sciences cognitives devrait mettre à jour le fonctionnement des processus mentaux et permettre leur classification. Cette vision optimiste des progrès de la connaissance n'est cependant pas partagée par Knight, qui tourne ici le dos au maître de la statistique descriptive anglaise et témoigne, en matière épistémologique, d'une influence plus méconnue.

Nous voilà parvenus aux frontières de la terra incognita : l'incertitude. Contre Pearson, Knight se trouve avoir recours à l'ennemi déclaré du professeur anglais ${ }^{68}$, le physiologiste allemand Du Bois-Reymond, le père de $l^{\prime}$ « agnosticisme ${ }^{69} »$. Ainsi Knight écrit-il : «L'auteur est en fait un empiriste radical en matière logique, ce qui signifie, pour autant que le raisonnement théorique est en cause, un agnostique pour toutes les questions dépassant les faits immédiats de l'expérience ${ }^{70}$. »

Puisque Knight évoque ici une polémique ancienne, il convient peut-être de discuter la question des influences dont il témoigne en matière de théorie de la connaissance. Tirant prétexte d'allusions à Henri Bergson ou William James, certains commentateurs ont conclu que Knight endossait leur épistémologie ${ }^{71}$. Quelle que soit par ailleurs sa sympathie pour divers aspects de la pensée de ces philosophes, il faut rappeler que c'est Knight

66. KNIGHT, 1921, p. 226.

67. KNight, 1921, p. 229 : «Nous ne pouvons étendre notre enquête au point de présenter toutes les raisons par lesquelles les hommes, fussent-ils cultivés, prennent leurs décisions, car nous ferions alors l'étalage d'un compendium de superstitions. »

68. Pearson, 1911, p. 26.

69. Voir Vidoni, 1988, pour une présentation de l'œuvre dans son contexte. Il semble curieux que Eмmetт, 1992, p. 33, ait choisi de qualifier Knight (comme au demeurant Ross, 1994, p. 9) de «moderniste agnostique », en référence au postmodernisme actuel. Ce faisant, il ne cite évidemment pas Du Bois-Reymond, alors que le genre prochain du modernisme de Knight semble mieux défini, à notre avis, par les termes mêmes de l'encyclique du pape Pie X, Pascendi Domini Gregis (\$ 2), en 1907.

70. KNIGHT, 1921, p. 201. À cette même page, Knight se réfère explicitement à Du BoIsREYMOND, 1872 et 1880.

71. MCKinNEy, 1977, est un exemple particulièrement frappant d'une attitude dont TeIRA SERrano, 1997, fait le bilan. 
lui-même qui met en garde son lecteur sur leurs contradictions propres. De plus, si Bergson ou James sont connus pour leur point de vue parfois spiritualiste sur l' « insondable mystère de l'esprit », il est clair que Knight est d'un autre avis :

«Les limites pratiques de la connaissance doivent cependant être imputées à des causes bien différentes [de celles suggérées par Bergson et James]. L'univers peut ne pas être entièrement connaissable (nous ne parlons bien sûr que des phénomènes objectifs, et non des problèmes qui transcendent l'expérience ordinaire), mais il est certainement connaissable dans une mesure qui dépasse si largement notre connaissance actuelle, que les limites de la connaissance dues aux incohérences du monde lui-même peuvent être négligées ${ }^{72}$. 》

Existe-t-il d'autres limites à la connaissance du processus de formation de nos estimations? Le dilemme que Knight expose dans l'introduction du chapitre VII de Risk, uncertainy and profit est le suivant : une explication scientifique rigoureuse de ces processus psychologiques doit être mécaniste, c'est-à-dire fondée sur une étude causale sans raisonnements finalistes. Cependant, l'explication subjective du comportement semble requérir un recours aux concepts de fin et de moyen qui induisent une téléologie :

«L'aptitude de la science à expliquer mécaniquement les réactions à des causes précédentes pourrait être assurée. Il n'en demeure pas moins que le sens commun se représente systématiquement les réactions comme conséquences d'une représentation consciente d'une situation future. Le rôle de la conscience est de donner à l'être la "connaissance" de l'avenir ${ }^{73}$. »

Il faut donc réconcilier ce double choix du mécanisme scientifique et de la téléologie vulgaire si l'on veut fonder la notion d'incertitude (au prix d'un éloignement de Pearson). Si la téléologie pouvait être réduite de façon mécaniste, alors les estimations préalables à l'action pourraient être réduites à des phénomènes cérébraux, lesquels pourraient être classifiés. Par contre, si les deux approches sont en fait inconciliables, cela signifie que l'incertitude existe.

Du Bois-Reymond était un disciple de l'école berlinoise de physiologie établie par Johannes Müller; il a lui-même contribué de façon significative au développement de la neurologie, en donnant une description mécaniste de l'activité nerveuse. Comme presque tous les membres de cette école, il partageait l'engagement positiviste. Par là, il entendait la possibilité d'une connaissance scientifique fondée sur l'observation empirique de mécanismes que l'on pouvait analyser intégralement en termes de matière et de

72. KNIGHT, 1921, p. 210.

73. KNIGHT, 1921, p. 201. 
force. Mais en 1872, Du Bois-Reymond donna une conférence à Leipzig dont la réputation vient de ce que la question de la possibilité d'une science entièrement positive y est posée. En effet, les concepts de matière et de force eux-mêmes sont inobservables, et donc irréductibles à l'empirie :

"On ne saura jamais mieux que maintenant ce qui, comme aimait à le dire Paul Erman, "ici", où est la matière, "hante l'espace". Même l'intelligence de Laplace, si infiniment supérieure à la nôtre, ne serait pas sur ce sujet plus perspicace que nous. Et c'est avec tristesse que nous constatons que nous avons atteint ici une des limites de notre esprit ${ }^{74}$.»

Suivant Du Bois-Reymond, il existe également une deuxième limite au pouvoir de l'esprit (et donc à la réalisation du programme positiviste) : la conscience. Évidemment, nous connaissons quelque chose des caractères cérébraux de l'activité de la conscience, mais comment passer de la matière en mouvement à l'esprit?

«On ne peut apprendre que sur certaines conditions internes du fonctionnement de l'intellect, qui sont rendues en quelque sorte équivalentes aux conditions extérieures via les impressions sensorielles; en revanche on ne peut rien savoir de la réalisation d'un état de l'intellect à travers ces conditions ${ }^{75}$.»

Les limites que présente Du Bois-Reymond pourraient être dépassées par une approche surnaturelle des phénomènes, mais dans la mesure où l'auteur se présente comme un athée militant, il ne peut proposer de suivre une telle voie. C'est pourquoi la conférence se termine par le fameux ignorabimus! : il reste des phénomènes que nous ne pourrons jamais connaître, quel que soit le progrès futur de la science.

Aussi étrange que cela puisse paraître, après vingt ans de vogue des sciences cognitives et de l'intelligence artificielle, l'incertitude knightienne apparaît comme une application de l'ignorabimus à ce que l'on peut appeler la «psychologie statistique » pearsonnienne :

«En dépit des déclarations enflammées de quelques zélotes sur la nouvelle science du "comportement", il semble invraisemblable que celle-ci puisse un jour remplacer la psychologie (qui est quelque chose de très différent) ou la théorie de la connaissance, en tout cas pour ce qui est de leurs formes historiques $^{76}$.»

Le seul fondement positif de la théorie de l'estimation subjective est la connaissance des mécanismes cérébraux et de leur relation avec les phéno-

74. Du Bois-Reymond, 1872, p. 62.

75. Du Bois-Reymond, 1872, p. 71.

76. KNIGHT, 1921, p. 203. 
mènes conscients ou intuitifs en jeu dans l'estimation. Ces phénomènes eux-mêmes se rapportent à la conscience d'une situation future et non pas aux données immédiates (empiriques) de la situation présente. Dans la mesure où les interactions entre esprit et cerveau demeureront dans l'ombre, même pour la science positive, il n'existera jamais de moyen de classement objectivement fondé, ce qui interdit une analyse en termes de probabilités statistiques.

« [I]l est toujours possible, du moins en théorie, d'ignorer la forme de la relation consciente et d'interpréter une réaction comme un effet mécanique d'une cause actuellement présente. Il n'en demeure pas moins que dans la pratique, c'est la situation présente à la conscience (et non celle qui est physiquement présente) que nous considérerons comme la cause qui mérite d'être étudiée ${ }^{77}$. »

C'est à cause de cet écart entre intériorité de la conscience et situation extérieure qu'il y a de l'incertitude et qu'il y en aura toujours : les estimations peuvent s'avérer fausses ${ }^{78}$, et cette fausseté (potentielle) même permet au profit d'apparaître. Le profit est ce que gagnent ceux qui ont mieux prévu que les autres : «[L]a capacité de former des jugements corrects [...] est la qualité la plus remarquable dans les affaires : c'est une activité tout à fait particulière, celle qui justifie au plus haut point la perception d'un salaire $^{79}$. » Le salaire nous ramène donc de la théorie de la connaissance à la théorie économique, ou du moins à ses fondements éthiques. Il est donc souhaitable d'indiquer comment le détour philosophique permet à Knight d'innover en matière de répartition.

LES CONSÉQUENCES ÉCONOMIQUES DE FRANK KNIGHT

La position épistémologique de Knight conduit à deux séries de conséquences. Une première série concerne la théorie de la répartition, telle que nous l'avons présentée au début de cet article. Dans la mesure où la question de la répartition a dominé les débats théoriques du début du siècle, il n'est pas très étonnant que la seconde série de conséquences touche à la conception même de la science économique et à la définition de son objet.

On peut donc interpréter la synthèse knightienne comme un essai de conclusion à la polémique du Quarterly Journal of Economics. Bien que

77. KNIGHT, 1921, p. 203.

78. KNIGHT, 1921, p. 201-202.

79. KNIGHT, 1921, p. 229. 
cela ne soit pas clairement affirmé par Knight, il est cependant possible de distinguer une lecture minimale - quoique déjà fortement novatrice d'une lecture radicale.

La lecture minimale constate que le profit de l'entrepreneur est la rémunération d'une incertitude (une situation où personne ne connaît les probabilités objectives). Dans la mesure où cette incertitude n'est pas mesurable a priori ni d'ailleurs a posteriori (on n'apprend rien d'une variable aléatoire par un tirage unique), il est impossible d'évaluer la vraie contribution de l'entrepreneur à la production. De ce fait, il est illusoire de chercher une quelconque «justice» dans la rémunération des entrepreneurs. En continuant le même raisonnement, on doit constater que l'incertitude condamne également la méthode de la productivité marginale qui semblait tout de même un peu trop simple. La valeur du produit dépend de la capacité de l'entrepreneur à le vendre. Il est donc impossible de mesurer même la productivité des autres facteurs. Exit donc la question de la justice de la répartition, de l'adéquation entre production et revenu. La détermination pratique en est impossible. La théorie économique de la répartition ne peut donc pas répondre aux questions éthiques.

L'abandon de la problématique de la répartition dans un cadre dynamique représente une nouveauté par rapport au point de vue de Clark qui, pour des raisons techniques, avait dû se situer dans un cadre statique. Knight insiste en fait sur la distance qui sépare « les conditions de la théorie de celles des faits ${ }^{80} »$, afin de justifier l'expulsion des problèmes éthiques. Dès le chapitre Iv, qui traitait de la répartition statique, l'auteur avait déjà critiqué point par point la théorie marginaliste, en insistant sur le caractère fallacieux des raisonnements à la marge et sur l'hétérogénéité des facteurs. Le premier point ne présente malheureusement pas grand intérêt car l'opposition résolue de Knight aux mathématiques l'oblige à de longs discours ennuyeux pour développer des arguments qui tiennent en une phrase (les fonctions de production ne sont pas toujours homogènes, donc la méthode de Clark ne peut s'appliquer toujours). Quant au second point, il renvoie évidemment à la «multiplication » des facteurs déjà aperçue à la fin de la polémique du Quarterly Journal of Economics. Cela conduit l'auteur à constater l'impossibilité d'attribuer, même dans une analyse statique, une part du produit à un facteur donné, et de terminer ainsi :

«La seule conclusion qui en résulte pour la politique sociale que nous insérerons ici est l'insistance sur la nécessité de se débarrasser de l'idée selon laquelle parce qu'un revenu est "gagné" il est "mérité" et non le contraire. Nous nous sommes en fait éloigné de ce principe dans la pratique, comme le montre [...] l'assistance aux infortunés et aux handicapés. Si l'on veut mainte-

80. KNIGHT, 1921, p. 30. 
nir le niveau d'humanité et de vie dans une société organisée, il faut choisir d'éliminer complètement la faiblesse ou bien d'imposer à la force le fardeau que la faiblesse ne peut porter $^{81}$.»

L'épistémologie de Knight se dévoile ici plus complètement : si la problématique en terme de droit au produit repose sur la seule contribution de chacun, alors il faut éliminer les inaptes. Sinon, il faut accepter de prélever sur le revenu des facteurs pour aider ceux qui ne peuvent prendre part à la production dans une mesure qui assurerait leur subsistance. Évidemment, ce n'est pas à la science économique d'indiquer comment choisir dans cette alternative, bien qu'elle puisse ensuite présenter les modalités du second choix.

La première lecture de Knight justifie donc l'abandon par la science économique de la problématique de la répartition, mais l'incertitude joue somme toute un rôle mineur dans cet abandon. C'est l'argument final, réservé à ceux que les considérations statiques n'avaient pas convaincus. Il est étonnant de constater qu'au même moment, des raisons similaires sont invoquées pour justifier un même mouvement. La même année 1921 voit la parution d'Une introduction à l'histoire industrielle de l'Angleterre d'Abbot Usher ${ }^{82}$. Usher y développe une étonnante thèse sur l'incertitude passée de la répartition:

«Il n'y a pas de question plus intéressante pour le lecteur moyen de l'histoire économique que le niveau de vie relatif des classes inférieures aux différentes périodes historiques. Beaucoup semblent croire que l'histoire économique n'apporte pas de contribution à la connaissance sans que quelques conclusions soient possibles sur le bien-être social. En général, aucune réponse satisfaisante n'est possible. Le matériel statistique est trop pauvre $[\ldots]^{83}$.»

Il serait bien évidemment absurde de comparer l'incertitude knightienne au manque de statistiques passées dont parle Usher. En revanche, il est étonnant de constater la parution en cette même année de deux ouvrages qui tournent délibérément le dos à la problématique économique des années 1890, en affirmant que la question de la justice de la répartition est indécidable par la science.

On le voit, lecture minimale ne signifie pas, loin s'en faut, absence de renouvellement de la problématique. Il s'agit simplement de montrer une conséquence logique de la pensée de Knight. La thèse radicale s'appuie pour sa part sur l'opposition entre Knight et Hawley, si on accepte de la mener à sa conséquence ultime. Il convient d'interpréter complètement le

81. KNIGHT, 1921, p. 129.

82. USHER, 1921.

83. UsHeR, 1921, p. 499. 
refus par Knight de se prononcer sur la distinction entre spéculateur et entrepreneur. Si Knight considère que Hawley a tort de penser que les spéculateurs gagnants doivent ce gain à leur «connaissance» et non à la «chance», il faut être cohérent. La spéculation n'est alors qu'une pure affaire de chance. De fait, c'est un jeu à somme nulle, et la fortune des uns constitue la ruine des autres. La seule différence avec la vie des affaires industrielles, c'est que le jeu, dans ce cas, est à somme positive. D'où vient alors que l'entreprise, contrairement à la spéculation, ne soit pas un jeu à somme nulle? De deux choses l'une : ou bien Knight est forcé de repartir sur les terrains glissants où Hawley s'était aventuré - l'impossible distinction du spéculateur et de l'entrepreneur - ou bien il admet que la somme positive provient d'ailleurs... Du travail, par exemple. Autant dire que Knight, avec l'incertitude, dissimule habilement le problème de l'origine du profit, sans le résoudre ${ }^{84}$.

Il apparait donc que Knight propose d'abandonner les considérations de justice sociale parce qu'elles sont non seulement entachées de jugements de valeur mais, de surcroît, indécidables. En positiviste, il juge évidemment sans équivoque une discipline dont l'objet se confondrait avec des jugements de valeur. Cependant, puisque c'est à l'objet même de la science, telle qu'elle est pratiquée avant lui, que Knight décide de renoncer, il se doit d'offrir une voie nouvelle pour cette discipline qui reste la sienne. Ainsi s'ouvre donc la deuxième série de conséquences : une nouvelle définition de l'économie, entendue comme développement du postulat de rationalité.

Depuis la fondation de l'économie politique, il était courant d'en appeler à la raison des acteurs pour justifier leurs arbitrages, leur exigence de primes de risque, ou, pour résumer : le fait qu'ils préfèrent plus à moins et ne fassent de cadeaux à personne. Ce «degré zéro de la rationalité », ce bon sens économique semblait si évidemment «la chose du monde la mieux partagée ${ }^{85}$, qu'elle constituait une donnée immédiate de la pensée économique. À partir des années vingt, les auteurs court-circuitent fréquemment les raisonnements à la marge, les arguments d'arbitrage, bref, les manifestations de ce «bon sens » pour en appeler au concept de « rationalité » en tant que telle. Ce tournant est déjà sensible chez Frank Knight,

84. En 1929, KNiGHT, 1929, p. 10, est un peu plus explicite quand il écrit : « La classification conventionnelle des facteurs de production distingue trois catégories : la terre, le travail, le capital ou si l'on préfère la nature, l'homme, l'attente. D'un point de vue éthique il serait plus approprié d'imputer le revenu à l'une des trois sources possibles que sont le libre choix ou effort, l'héritage et la chance. La chance est la source la plus importante des trois! Le principe d'égalité dans la liberté, tel qu'il s'exprime dans un ordre social fondé sur le libreéchange, ne nous conduit nulle part en ce qui concerne la justice sociale. Tout dépend de ce que les individus apportent dans l'échange, et ceci est de l'ordre des faits, pas de l'éthique. »

85. Descartes, 1637, p. 126. C'est la première phrase de ce traité. 
Lionel Robbins ou Paul Samuelson. Très rapidement, la nature de cette rationalité qui apparaît alors comme abstraction devient un objet d'étude (en témoigne par exemple, à la fin des années trente, la controverse sur la fonction d'utilité). La problématique et les méthodes de l'économie politique classique, l'étude de la production et de la répartition sociale, sont alors concurrencées par un nouveau paradigme : la recherche sur les modalités de la rationalité des individus.

Dans cette quête nouvelle, le risque, critérium de la décision incertaine, fournit un objet de choix pour l'étude de la rationalité. Les travaux sur le sujet sont encore marginaux avant-guerre. C'est au cours de la Seconde Guerre mondiale et dans les années qui vont suivre, à l'occasion d'une rencontre entre des mathématiciens européens exilés et les jeunes économistes américains, que la question du risque va prendre une ampleur nouvelle. Il convient de préciser comment la naissance de la théorie de la décision s'accompagne de la reconstruction de la théorie économique comme science des choix. On pourrait être tenté de croire que Léon Walras, inquiet de construire une science rationnelle, anticipait largement sur Knight ou Robbins. Il va être démontré d'abord que ce n'est pas le cas. Le projet inachevé de Knight en matière d'économie rationnelle sera présenté ensuite, et en dernier lieu, son accomplissement chez ses lecteurs et partisans anglais à la London School of Economics (LSE).

L'idée d'étudier les décisions rationnelles ne semble donc pas à proprement parler une nouveauté : l'influence de David Hume sur Smith et la tradition classique anglaise a laissé une profonde empreinte ${ }^{86}$. Si ces références trop philosophiques semblent également trop lointaines, on peut observer que l'école de Lausanne offrit également une perspective sur la rationalité. Quelle est la position de ces auteurs?

Walras emploie explicitement l'épithète « rationnel » pour caractériser certains comportements des agents : il parle ainsi de «comptabilité rationnelle ${ }^{87} »$. Mais ailleurs, Walras fait usage d'un sens différent de la même épithète : «L'économie politique est, comme l'astronomie, comme la mécanique, une science à la fois expérimentale et rationnelle ${ }^{88} »$. Ici, l'attribut ne peut plus recevoir une interprétation en terme de rationalité économique, il semble qu'il faille entendre quelque chose comme la mécanique rationnelle. D'après André Lalande, cette formule renvoie à «l'ensemble

86. Picavet, 1996 , p. 53-70.

87. WALRAS, 1874 , p. $284-285$ : «J'estime que, pour tenir une comptabilité rationnelle, un entrepreneur qui est propriétaire du sol qu'il exploite ou qu'il occupe, qui participe à la direction de son entreprise, qui a des fonds engagés dans l'affaire, doit débiter ses frais généraux et se créditer lui-même d'un fermage, d'un salaire et d'un intérêt calculés aux taux du marché des services producteurs et au moyen desquels il subsiste, sans faire à la rigueur, comme entrepreneur, ni bénéfice ni perte. »

88. WalRAs, 1900, p. 22. Nous soulignons. 
de toutes les questions de mécanique qui sont traitées par une méthode purement déductive à partir des notions de masse, de force, de liaison et d'inertie $^{89} \gg$. L'adjectif rationnel caractérise donc une conception de la connaissance scientifique, dont le modèle est donné par les mathématiques elles-mêmes. En effet, Walras écrit :

«La méthode mathématique n'est pas la méthode expérimentale, c'est la méthode rationnelle. Les sciences naturelles proprement dites se bornent-elles à décrire purement et simplement la nature et ne sortent-elles pas de l'expérience? Je laisse aux naturalistes le soin de répondre à cette question. Ce qui est sûr, c'est que les sciences physico-mathématiques, comme les sciences mathématiques proprement dites, sortent de l'expérience dès qu'elles lui ont emprunté leurs types. Elles abstraient de ces types réels des types idéaux qu'elles définissent; et, sur la base de ces définitions, elles bâtissent $a$ priori tout l'échafaudage de leurs théorèmes et de leurs démonstrations. Elles rentrent, après cela, dans l'expérience non pour confirmer, mais pour appliquer leurs conclusions ${ }^{90}$. »

Cette longue citation constitue la définition par Walras de l'adjectif rationnel; elle résonne particulièrement avec celle de Lalande, bien que l'économiste porte l'accent sur les mathématiques. Est rationnel ce qui emprunte sa structure aux mathématiques, ce qui peut être expliqué par les mathématiques (les travaux des ingénieurs économistes de l'entre-deuxguerres, comme Maurice Bouytaud, François Divisia, Georges et Édouard Guillaume, portent encore la trace de cette analogie). Dès lors, parler de rationalité des individus ne peut constituer qu'un abus de langage, même si des comportements se révèlent parfois rationnels.

Vilfredo Pareto poursuit cette analogie entre mécanique rationnelle et économie pure ${ }^{91}$, mais crée sa propre terminologie, sans renoncer à la référence mathématique. Pareto introduit en effet la notion d'action logique (la logique étant une branche des mathématiques depuis les travaux de George Boole), et l'utilise ainsi :

«[...] nous ne considérons que des actions qui se répètent, ce qui nous permet d'admettre que c'est un lien logique qui unit ces actions. Un homme qui, pour la première fois, achète un certain aliment pourra en acheter plus qu'il ne lui en faut pour satisfaire ses goûts, en tenant compte du prix; mais à un second achat

89. LaLANDE, 1947 , p. 890.

90. Walras, 1874, p. 53.

91. Ce sont les termes de PARETo, 1898, voir p. 106: «L'économie pure emploie les mêmes procédés que la mécanique rationnelle », ou p. 109 : «En mécanique, après la statique, nous considérons la dynamique. Il est de même en économie pure. Et, pour le dire en passant, c'est la voie qui conduit à donner des crises économiques la seule explication qui soit rationnelle.» Nous soulignons. 
il rectifiera son erreur, en partie du moins; et petit à petit, il finira par se procurer exactement ce dont il a besoin. Nous le considérons au moment où il est arrivé à cet état. De même, s'il se trompe une première fois dans ses raisonnements au sujet de ce qu'il désire, il les rectifiera en les répétant, et il finira par les rendre complètement logiques ${ }^{92}$. »

L'économie pure représente donc les individus « au moment où ils sont arrivés à cet état », c'est-à-dire lorsque leurs actions ont acquis une « logique », lorsqu'elles sont cohérentes. Il faut observer que Pareto fait des actions logiques, et non de la logique elle-même, l'objet de la science.

Il apparaît alors que si Walras et Pareto postulent la cohérence des agents, ils n'en font pas pour autant l'objet d'une théorie particulière, et encore moins l'objet de la science économique. Pour ces auteurs, l'hypothèse de la décision rationnelle n'est somme toute qu'un des moyens de la démarche scientifique. D'un autre côté, Walras et Pareto restent très proches de la problématique de la justice sociale; comme en témoignent les travaux walrasiens d'économie appliquée ou les tentatives parétiennes de s'opposer à l'influence marxiste. La part positive du programme de l'école de Lausanne semble cependant avoir inspiré Knight, qui va pousser plus avant.

Parmi les conséquences de l'engouement des historiens de la pensée économique pour Knight, on peut également citer des travaux récents sur la méthodologie du maître de Chicago. Ces articles montrent généralement Knight comme un défenseur de l'hypothèse de maximisation ${ }^{93}$, accessoirement (ou à titre de corollaire) comme un proche de Robbins ${ }^{94}$. Ces deux caractérisations expriment autant de modalités d'adhésion au projet de construire une science des choix. Knight ne va cependant pas jusqu'au bout du mouvement qu'il a contribué à initier; l'étude de ces hésitations permettra de comprendre la spécificité de ses partisans anglais.

S'il ne considérait pas l'économie mathématique comme un «culte» ouvert aux seuls « initiés ${ }^{95}$, Knight pourrait apparaître comme un continuateur de Pareto. En effet, de même que Pareto restreint le champ de l'économie pure aux actions logiques, Knight cantonne l'analyse économique aux actes conformes aux fins :

"On peut dire avec raison que l'analyse économique s'intéresse à la "conduite", dans le sens spencérien d'actes conformes aux fins, ou de conformité des actes aux fins, comme cas particulier de la catégorie plus large de “comportement" en général ${ }^{96}$. »

92. Pareto, 1906, p. 145-146.

93. ЕмMETT, 1994, p. $277-278$

94. HAMmOND, 1991, p. 368.

95. KNIGHT, 1921, p. 14.

96. KNIGHT, 1921, p. 52. 
En particulier, Knight s'accorde avec Pareto sur un point crucial : celui du champ de l'économie, qui n'a pas à se soucier de rendre compte de toutes les actions liées à la monnaie, à la production, mais seulement de certaines actions caractérisées par leur logique interne. De ce fait, la « sphère économique »n'est plus un domaine spécifique du social : est économique ce qui relève d'un type d'action (l'action rationnelle) et non d'un objet donné (l'échange).

En lisant plus avant Knight, il semble que son exposition du postulat de rationalité représente un nouveau pas par rapport aux revendications logicistes de Pareto:

«Nous faisons l'hypothèse que les membres du corps social agissent avec une "rationalité" complète. Nous n'entendons pas par ces termes qu'ils soient, "comme des anges, distinguant le bien et le mal"; nous ne faisons pas d'hypothèses particulières sur les motivations des individus [...]; nous les supposons simplement capables de "savoir ce qu'ils veulent" et de le rechercher "intelligemment". Leur comportement est entièrement une "conduite" dans le sens où nous l'avons préalablement défini; leurs actes répondent à des stimulations réelles, conscientes, stables et cohérentes; rien n'est l'effet du caprice ou de la volonté d'expérimenter de façon désordonnée, tout est délibéré. Ils sont supposés connaître absolument les conséquences de leurs actes au moment où ceux-ci sont réalisés, et de les réaliser en connaissance de cause ${ }^{97}$. »

Une différence fondamentale apparaît entre Pareto et Knight. Chez Pareto - comme chez Walras au reste —, seules les actions étaient logiques ou rationnelles, il ne semblait pas opportun ni intéressant de qualifier les sujets des actions. Knight définit au contraire des sujets rationnels. Ce faisant, il développe la notion de rationalité, essentiellement comme conscience des fins et des moyens («tout est délibéré »), mais aussi comme cohérence (à travers la notion de «conduite»).

La question de savoir si Knight s'accorde ou non avec l'école de Lausanne, ou, plus généralement, avec les diverses tentatives de définition de l'homo œconomicus, est justement celle que traitent les études récentes sur son épistémologie. Les auteurs de ces travaux dressent, si besoin était, le portrait d'un homme hésitant. D'une part, Daniel Hammond montre la « tendance de Knight à déclarer simultanément, ce qui est contradictoire, que l'économie est une science et qu'elle n'en est pas une ${ }^{98}{ }^{\prime}$. L'idée fondamentale de cet auteur est que Knight est coincé entre le désir de l'analogie avec la physique (telle qu'elle est exposée au premier chapitre de Risk,

97. KNIGHT, 1921, p. 76-77. Nous soulignons.

98. HAMmOND, 1991, p. 377. 
uncertainty and profit, et réaffirmée tout au long de l'œuvre de Knight) et la volonté de construire une science hypothético-déductive qui ne soit pas soumise à la sanction de l'expérience. Ce dernier point est qualifié par Hammond, en référence au cercle de Vienne, d' ' antipositivisme ». D'autre part, Ross Emmett montre que l'hypothèse de rationalité (ou de maximisation, comme il l'indique), qui permet précisément d'édifier la science déductive, ne permet d'expliquer qu'assez peu de phénomènes : « La vision scientifique de la vie est limitée et partielle ${ }^{99}$. $\gg$ Nous nous contentons de souscrire au point de vue de ces deux auteurs; en nous permettant d'ajouter une perspective historique qui confirme le caractère hésitant de Knight. Ce dernier propose bien d'avancer d'un pas de plus dans la construction de la science des choix, mais il restreint explicitement, au point de se montrer lui-même déçu, la portée d'un tel projet.

Knight ne vaut cependant pas seulement par la lecture que nous pouvons faire aujourd'hui de ses écrits, il est manifeste qu'il a profondément marqué les membres du Robbins' group, dont la contribution à la constitution de la science des choix est particulièrement éminente.

En 1932, Lionel Robbins propose une nouvelle définition de l'économie qui, comme le fait remarquer Peter Groenwegen, « anéantit (destroyed) la conception que les économistes classiques avaient de leur science ${ }^{100} \gg$. En effet, l'économie devient «la science qui étudie les comportements humains comme une relation entre des fins et des moyens rares et susceptibles d'emplois alternatifs ${ }^{101} »$. Il n'existe donc plus dans cette optique une "sphère économique », partie de la vie sociale distincte de la vie politique, car tous les choix soumis à des contraintes peuvent être réputés économiques, comme le laisse penser le premier chapitre de l'Essai de Robbins. On peut être tenté de réduire l'écart avec les définitions de l'économie antérieures, en particulier avec celle de Walras, en montrant que la «richesse sociale ${ }^{102}$ » de ce dernier n'est plus à proprement parler une ploutologie, et même qu'elle fait apparaître la notion de rareté centrale chez Robbins. De même, on pourrait montrer que l'étude de l'économie rationnelle, des actions logiques, ou des conduites «dans le sens spencérien » ne sont pas incompatibles avec le programme de Robbins. On peut ainsi continuer à chercher les points de contact entre cette définition de l'économie et les programmes de recherche antérieurs, mais il faut concéder que, si l'objet attribué à l'économie par Robbins n'est pas nouveau, ce

99. Knight cité par Емметт, 1994, p. 285.

100. Groenwegen, 1987, p. 906.

101. RobBins, 1932, p. 116.

102. WaLras, 1900, p. xxI, désigne ainsi l'ensemble des choses rares, «c'est-à-dire qui, d'une part, nous sont utiles, et qui, d'autre part, n'existent à notre disposition qu'en quantité limitée ». 
qui est nouveau, c'est d'en faire l'objet unique de l'économie. À partir de là, il n'est peut-être pas inutile de se demander quel est le rapport entre cette nouvelle définition de l'économie et l'impasse de la théorie de la répartition que montre Knight. Au départ, il n'y a qu'une simple coïncidence temporelle. Après une étude de l'emploi du temps à la LSE en 1928-1930, et en particulier des occupations de John Hicks, il semble que Knight ait exercé une influence directe sur la formation du programme de Robbins.

Knight ne s'est pas rendu personnellement à Londres dans les années vingt, en revanche Stigler nous apprend que son livre était une référence de base à la LSE dès le début des années trente ${ }^{103}$. Le fait de réserver, en 1929, une place d'honneur à un économiste américain à Londres appelle quelque explication. Charles Blitch fournit un élément important pour comprendre la diffusion de l'ouvrage de Knight: la venue en Europe d'Allyn Young, le directeur de thèse de Knight.

Young se rend sur le Vieux Continent en tant qu'expert nommé par le gouvernement américain pour y négocier le traité de Versailles, puis pour mettre sur pied un plan qui porte son nom ${ }^{104}$. À la suite de cet épisode, Young est nommé par William Henry Beveridge à la chaire d'économie politique de la LSE pour la période 1927-1929. Blitch, qui rapporte ces faits, s'étonne que, dans le désarroi suscité par la démarche sraffienne ${ }^{105}$, une contribution de Young ${ }^{106}$ à la théorie de la répartition qui permettait d'échapper à certains problèmes soulevés par Piero Sraffa n'ait pas rencontré un plus grand succès. En fait, les collègues anglais de Young à la LSE ont été plus intéressés par le livre que ce dernier apportait avec lui que par les considérations néo-smithiennes de ce haut fonctionnaire américain. Car c'est très vraisemblablement Young qui a fait connaître l'ouvrage de Knight à la LSE : Hicks situe en 1929 les séances du Robbins' group (réunissant Roy Allen, Nicholas Kaldor, Abba Lerner, Lionel Robbins, luimême et d'autres) consacrées à la lecture de Risk, uncertainty and profit ${ }^{107}$. Or il apparaît que Hicks en a tiré rapidement des conclusions qui vont parfaitement dans le sens souhaité par Robbins.

Peu de temps après la lecture de Knight par le Robbins' group, Hicks publie une «Théorie de l'incertitude et du profit ${ }^{108}$ ». Il y discute méticuleusement les thèses du "professeur Knight », qui est cité presque à chaque page. De façon générale, si l'Anglais tient à se distinguer de l'Amé-

103. STigler, 1987, p. 56.

104. Blitch, 1983, p. 6 sqq.

105. SRAFFA, 1926, suscite une nouvelle polémique sur la répartition.

106. Young, 1928.

107. Hicks, 1977, p. 135.

108. Hicks, 1931. 
ricain, ce n'est que par des nuances : ainsi, la moitié de la p. 175 (en note), est consacrée à discuter la nécessité d'employer l'expression « réduction des risques » plutôt qu' "élimination» comme le fait Knight. Si, dans le détail, Hicks ne semble pas s'éloigner beaucoup de Knight, il lui semble également fidèle dans la lecture du problème de la répartition; à ceci près qu'il en tire des conclusions inattendues.

Comme Knight, Hicks prend acte de l'impossibilité de mesurer à l'avance la part du revenu revenant au groupe des «capitaines d'industrie ». Il conclut que ce problème est mineur en comparaison de la "possibilité que les affaires d'un groupe assez important et homogène pour représenter un intérêt économique, puisse manquer à l'indépendance ${ }^{109}{ }$. À l'abri des circonlocutions, Hicks prend conscience du fait que l'impossibilité de mesurer (ex ante) le revenu de l'entrepreneur conduit à l'impossibilité de mesurer (ex ante) tous les revenus: on a beau considérer des groupes de gens pertinents (Hicks dit homogènes) pour appliquer la loi des grands nombres, rien n'y fait. La question de la répartition véritable est donc indécidable. D'où la conclusion de Hicks :

«On doit s'attendre [...] à ce que ceux qui retirent leur rémunération sous la forme d'un profit, reçoivent un paiement total qui, par comparaison avec les services rendus, soit à peine plus grand, et parfois moins, que ceux des investisseurs dans des affaires sûres ${ }^{110}$.»

Ce qui est une agréable manière d'écrire qu'on ne sait pas grand-chose. Ainsi, pourrait-on penser que Hicks parvient à la même impasse que Knight et s'applique également à la dissimuler. C'est le cas. Cependant, les écrits ultérieurs de Hicks parlent beaucoup plus clairement.

À la suite de cet article, l'Anglais décide tout bonnement de séparer les deux matières. D'une part, il produit une théorie de la répartition d'où le profit et le risque sont exclus ${ }^{111}$. D'autre part, il traite le choix risqué dans une série d'articles assez méconnus, mais qui représentent tout de même la première théorie économique de la décision. On continue donc à faire de l'économie ancienne manière en s'inscrivant dans le projet walrasien - et Hicks n'a pas peu fait pour publier le nom de Walras auprès de ses collègues anglo-saxons. Dans le même temps, on travaille explicitement dans le projet robbinsien de science des choix, en s'intéressant à des relations d'ordre avant même la polémique sur l'utilité. D'une certaine façon, ces travaux sont complémentaires : la reformulation parétienne de la fonction d'utilité par Hicks l'aide dans ses travaux sur le choix risqué. D'un autre

109. Hicks, 1931, p. 188.

110. Hicks, 1931, p. 188.

111. Hicks, 1932. 
côté, ils montrent que la transformation de l'économie en science des choix, si elle est inachevée, est tout de même avancée dès la publication de la deuxième édition de l'Essai de Robbins (1935).

Les économistes considèrent, jusqu'aux années 1930, le risque comme une «force distributive », un élément explicatif de la théorie de la répartition. Ce risque n'est jamais défini ni bien sûr quantifié (ce qui autoriserait une définition implicite), il sert exclusivement à l'édification d'une théorie normative, intéressée à la question de la justice sociale. Dans ce contexte, les prétentions d'un John Neville Keynes ${ }^{112}$ à l'édification d'une science positive semblent hors d'atteinte puisque l'élaboration théorique à proprement parler ne dépasse pas le stade d'une prénotion, et la visée de la théorie est si entièrement normative qu'elle ne permet pas de s'affranchir de jugements de valeur, qui constituent l'enjeu même de la science.

La démonstration par Knight du caractère non mesurable de l'incertitude économique coupe court à ce genre de questionnement, à l'impasse où menait la polémique du Quarterly Journal of Economics. La théorie du risque, bien loin d'entrer en crise, fournit alors l'instrument du relèvement de l'économie, entendue dès lors comme une théorie de la décision rationnelle et non plus comme une description du fonctionnement des économies soumise à des enjeux éthiques. Se profile donc le retour du «point de vue décisionnel » (présent chez Pascal, Laplace, qui s'épanouit à nouveau chez John von Neumann, Oskar Morgentern, Leonard Savage, etc.), après une «éclipse » que Georges Guilbaud remarquait au début des années cinquante ${ }^{113}$. Même si les héritiers de Knight (Friedman, Stigler et ses nombreux autres élèves à Chicago) ont assez vite eu raison de la répugnance de leur maître pour les mathématiques, c'est dans cette direction qu'ils ont poussé son positivisme porté peut-être jusqu'au scientisme, au culte de la formalisation pour elle-même ${ }^{114}$.

Pierre-Charles PRAdIER

David Teira Serrano (janvier 2000).

112. Keynes, 1890.

113. Guilbaud, 1954.

114. Friedman, 1953. 


\section{LISTE DES RÉFÉRENCES}

Armatte (Michel), 1995, Histoire du modèle linéaire, thèse, Paris, École des hautes études en sciences sociales.

Bernoulli (Daniel), 1731, « Specimen theoriae novae de mensura sortis », rééd. in Die Werke von Daniel Bernoulli, Bâle, Birkhäuser, 1982, t. II; trad. franç. par Raoul Charreton, notes de Bernard Bru, «Esquisse d'une nouvelle théorie de mesure du sort », Cahiers du séminaire d'histoire des mathématiques, vol. VI, 1984, p. 61-77.

Butch (Charles P.), 1983, «Allyn A. Young. A curious case of professional neglect », History of Political Economy, vol. XV, 1, p. 1-24.

BöHм-BAwerk (Eugen von), 1884, Kapital und Kapitalzins, Jena, Fischer; trad. angl. Capital and interest, Londres, Macmillan, 1890.

Bouytaud (Maurice), 1935, Essai d'économique rationnelle, Paris, GauthierVillars.

CARver (Thomas N.), 1901, «Clark's Distribution of wealth », Quarterly Journal of Economics, vol. XV, 4, p. 578-602.

Clark (John Bates), 1892, «Insurance and business profit », Quarterly Journal of Economics, vol. VII, 1, p. 40-54.

Clark (J. B.), 1899, The Distribution of wealth, New York, Macmillan; rééd. New York, A. M. Kelley, 1965.

Corry (Bernard A.), 1987, «Robbins, Lionel Charles », in John Eatwell et al., éd., The New Palgrave. A dictionary of economics, New York, Stockton Press, vol. IV, p. 206-208.

Debouzy (Marianne), 1972, Le Capitalisme «sauvage» aux États-Unis, Paris, Seuil.

Descartes (René), 1637, Discours de la méthode, Leyde, Jan Mayre; rééd. Paris, Gallimard, 1952.

Dewey (Donald), 1986, «Frank Knight before Cornell, some light on the dark years », in Warren J. SAmuELs, éd., Research in the history of economic thought and methodology, Greenwich, CT, JAI Press, vol. IV.

Divisia (François), 1927, Économique rationnelle, Paris, Doin.

Du Bois-Reymond (Emil), 1872, «Über die Grenzen des Naturerkennens », in Siegfried Wollgast, éd., Emil Du Bois-Reymond. Vorträge über Philosophie und Gesellschaft, Hambourg, Felix Meiner, 1974, p. 45-53.

Du Bois-Reymond (E.), 1880, «Die Sieben Welträtsel », in Siegfried Wollgast, éd., Emil Du Bois-Reymond. Vorträge über Philosophie und Gesellschaft, Hambourg, Felix Meiner, 1974, p. 159-189.

EDGEWORTH (Francis Ysidro), 1888, « The mathematical theory of banking », Journal of the Royal Statistical Society, vol. LI, p. 113-127.

Edgeworth (F. Y.), 1904, "The theory of distribution », Quarterly Journal of Economics, vol. XVIII, p. 159-219.

Edgeworth (F. Y.), 1996, Writings in probability, statistics and economics, Aldershot, Elgar, 3 vol. 
EMERY (Henry Crosby), 1898, « The place of the speculator in the theory of distribution ", Papers and proceedings of the twelth meeting of the American Economic Association, supplément annuel, American Economic Review, p. 103-118.

Emmetт (Ross B.), 1992, "Frank Knight on the conflict of values in economic science ", in Warren J. SAmuels, éd., Research in the history of economic thought and methodology, Greenwich, CT, JAI Press, vol. X, p. 87-103.

Eмmetт (R. B.), 1994, « Maximizers versus good sports. Frank Knight's curious understanding of exchange behavior », in Neil DE Marchi et Mary MoRgan, éd., Higgling. Transactors ant their markets in history of economics, $\mathrm{n}^{\circ} \mathrm{special}$ de History of Political Economy, vol. XXVI, p. 276-292.

Emmetт (R. B.), 1999, « The economist and the entrepreneur. Modernist impulses in Risk, uncertainty and profit », History of Political Economy, vol. XXXI, 1, p. 29-52.

FonTAINE (Philippe), 1999, «Classical political economy between two fires. JeanBaptiste Say and Frank H. Knight on the enterprise economy », History of Political Economy, vol. XXXI, 1, p. 1-28.

FRIEDMAN (Milton), 1953, "The methodology of positive economics », in ID., Essays in positive economics, Chicago, The University of Chicago Press; rééd. in Daniel Hausman, éd., The Philosophy of economics. An anthology, Cambridge, Cambridge University Press, 1984; trad. franç. Paris, Litec, 1995.

Groenwegen (Peter D.), 1987, "Political economy" and "economics" ", in John Eatwell et al., éd., The New Palgrave. A dictionary of economics, New York, Stockton Press, vol. III, p. 904-907.

Guerrien (Bernard), 1999, La Théorie économique néo-classique, Paris, La Découverte (Repères).

Guilbaud (Georges-Théodule), 1954, «Leçons sur les éléments principaux de la théorie mathématique des jeux », in Georges Th. Guilbaud, Pierre Massé et Robert HÉNon, Stratégies et décisions économiques, études théoriques et applications aux entreprises, Paris, Centre national de la recherche scientifique.

Guillaume (Georges et Édouard), 1932, Sur les fondements de l'économique rationnelle, Paris, Gauthier-Villars.

Guillaume (G. et É.), 1937, L'Économique rationnelle. De ses fondements aux problèmes actuels, Paris, Hermann.

Guillaume (G. et É.), 1938, «Controverse au sujet d'une nouvelle économique rationnelle. Réponse aux commentaires de M. F. Divisia », Revue d'économie politique, vol. LII, p. 1220-1223.

Hammond (J. Daniel), 1991, «Frank Knight's antipositivism », History of Political Economy, vol. XXXIII, 3, p. 359-380.

Hawley (Frederick B.), 1891, "The fundamental error of Kapital und Kapitalzins », Quarterly Journal of Economics, vol. VI, 3, p. 280-307.

Hawley (F. B.), 1893, « The risk theory of profit », Quarterly Journal of Economics, vol. VII, 4, p. 459-475.

Hawley (F. B.), 1900, «Enterprise and profit », Quarterly Journal of Economics, vol. XV, 1, p. 75-104.

HAYNES (John), 1895, «Risk as an economic factor », Quarterly Journal of Economics, vol. IX, 4, p. 409-449.

HICKS (John Richard), 1931, « The theory of uncertainty and profit », Economica, vol. IX, mai, p. 170-189.

Hicks (J. R.), 1932, Theory of wages, Oxford, Clarendon Press.

Hicks (J. R.), 1933, « Gleichgewicht und Konjonktur », Zeitschrift für Nationalökonomie, vol. L, p. 440-455.

Hicks (J. R.), 1977, Economic perspectives, Oxford, Clarendon Press. 
Ingrao (Bruna) et Israel (Giorgio), 1987, La Mano invisibile, Rome, Laterza; trad. angl. The Invisible Hand, Cambridge, MA, The MIT Press.

Julien (Claude) et Toinet (Marie-France), « A.F.L.-C.I.O. », in Encyclopaedia Universalis, 1992, vol. I, p. 386a-389b.

KEYNES (John Maynard), 1921, A treatise on probability, Londres, Macmillan; rééd. in The Collected Writings of J. M. Keynes, Londres, Macmillan, 1973, vol. VIII.

KEYNES (John Neville), 1890, The Scope and method of political economy, Londres, Macmillan; 4 e éd., rééd. New York, A. M. Kelley, 1986.

KNIGHT (Frank Hyneman), 1921, Risk, uncertainty and profit, Boston; rééd. New York, A. M. Kelley, 1964.

KNight (F. H.), 1929, "Freedom as fact and criterion», Journal of Ethics, vol. XXXIX, p. 129-147; repr. in KNIGHT, 1947, p. 1-18.

Knight (F. H.), 1935, The Ethics of competition and other essays, New York, Harper \& Brothers; rééd. New York, A. M. Kelley, 1951.

Knight (F. H.), 1947, Freedom and reform, New York, Londres, Harper \& Brothers.

LaLANDE (André), 1947, Vocabulaire technique et critique de la philosophie, Paris, Presses universitaires de France; rééd. Paris, Presses universitaires de France, 1993.

LANGLoIs (Richard N.) et Cosgel (Metin M.), 1993, « Frank Knight on risk, uncertainty and the firm », Economic Inquiry, vol. XXXI, p. 456-465.

LeRoy (Stephen F.) et Singell (Larry D.), 1987, « Knight on risk and uncertainty », Journal of Political Economy, vol. XCV, 2, p. 294-406.

Marchal (Jean) et LeCAILlon (Jacques), 1958, La Répartition du revenu national, Paris, Génin, 4 vol.

Marshall (Alfred), 1923, Money, credit \& commerce, Londres, Macmillan.

McKinney (John), 1976, «Frank Knight and Chicago libertarianism », in Warren J. SAmuels, éd., The Chicago School of political economy, New Brunswick, Transaction Publishers, p. 191-213.

MCKINNEY (J.), 1977, «Frank H. Knight on uncertainty and rational action », Southern Economic Journal, vol. XLIII, april, p. 1438-1452.

Morgan (Mary), 1994, "Marketplace morals and the american economists. The case of John Bates Clark », in Neil Marchi et Mary Morgan, éd., Higgling. Transactors ant their markets in history of economics, $\mathrm{n}^{\circ}$ spécial de History of Political Economy, vol. XXVI, p. 229-252.

Niehans (Jürg), 1991, «Böhm-Bawerk versus John Doe. The interest controversies », History of Political Economy, vol. XXIII, 4, p. 567-586.

PARETo (Vilfredo), 1898, «Comment se pose le problème de l'économie pure?» [Mémoire présenté en décembre 1898 à la société « Stella »], Lausanne, chez l'auteur, 1899; rééd. Cahiers Vilfredo Pareto, vol. I, 1963, p. 121-130.

Pareto (V.), 1906, Manuale di economia politica; trad. franç. rééd. in Giovano Busino, éd., Euvres complètes de Vilfredo Pareto, Genève, Droz, 1966, t. VIII.

Pearson (Karl), 1911, The Grammar of science, $3^{\mathrm{e}}$ éd. Londres, Adam and Charles

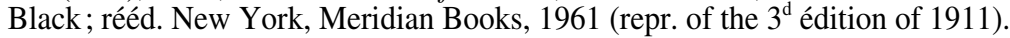

Picavet (Emmanuel), 1996, Choix rationnel et vie publique, Paris, Presses universitaires de France.

PRADIER (Pierre-Charles), 1998, Concepts et mesures du risque en théorie économique. Essai historique et critique, thèse, Cachan, École normale supérieure.

Ricardo (David), 1952, lettre à Malthus du 9 octobre 1820, in Works, Cambridge, Cambridge University Press, vol. VIII, p. 278-279.

RobBins (Lionel), 1932, An essay on the nature and significance of economic science, $2^{\mathrm{e}}$ éd. Londres, Macmillan, 1935 ; rééd. in Daniel Hausman, éd., The 
Philosophy of economics. An anthology, Cambridge, Cambridge University Press, 1984.

Ross (Dorothy), 1991, The Origins of American social science, Cambridge, Cambridge University Press.

Ross (D.), 1994, «Modernism reconsidered », in ID., Modernist impulses in the human sciences, 1890-1930, Baltimore, John Hopkins University Press.

SAmuelson (Paul Anthony), 1947, Foundations of economic analysis, Cambridge, Harvard University Press.

SMith (Adam), 1776, An inquiry into the nature and causes of the wealth of nations, Londres, W. Straham \& T. Cadell; rééd. Londres, Penguin, 1982; trad. franç. de Paulette TAÏ̈B, Paris, Presses universitaires de France, 1996.

SRAFFa (Piero), 1926, "The laws of return under competitive conditions », Economic Journal, vol. XXXVI, p. 535-550.

Stigler (George), 1971, Foreword to Knight, $1^{\text {re }}$ éd. 1921, ici rééd. Chicago, University of Chicago Press.

Stigler (G.), 1987, «Knight, Frank Hyneman », in John Eatwell et al., éd., The New Palgrave. A dictionary of economics, New York, Stockton Press, vol. III, p. $55-59$.

TAussig (Frank William), 1895, «The employer's place in distribution », Quarterly Journal of Economics, vol. X, 1, p. 67-94.

Teira Serrano (David), 1997, « Knight and Friedman on knowledge and chance. A perspective from nineteenth-century agnosticism », communication au colloque European conference on the history of economics (ECHE), Anvers, Universitaire Faculteiten Sint-Ignatius Antwerpen (UFSIA), avril 1997.

UsHer (Abbot Payson), 1921, An introduction to the industrial history of England, Londres, Harrap.

VÉRIN (Hélène), 1982, Entrepreneurs, entreprise. Histoire d'une idée, Paris, Presses universitaires de France.

Vidoni (Ferdinando), 1988, Ignorabimus! Emil Du Bois-Reymond e il dibattito sui limiti della conoscenza scientifica nell' Ottocento, Milan, Marcos \& Marcos.

WaLRAs (Léon), 1874, Éléments d'économie politique pure ou théorie de la richesse sociale, $1^{\mathrm{re}}$ éd. Lausanne, L. Corbaz; rééd. in Euvres économiques complètes, Paris, Economica, 1988, vol. VIII.

Walras (L.), 1900, Éléments d'économie politique pure ou théorie de la richesse sociale, $4^{\mathrm{e}}$ éd. Paris, Librairie générale de droit et de jurisprudence; rééd. in Euvres économiques complètes, Paris, Economica, 1988, vol. VIII.

Wick (Warner), 1973, « Frank Knight. Philosopher at large », Journal of Political Economy, vol. LXXXI, May-June, p. 513-515.

WicksteEd (Philip), 1894, Essay on the co-ordination of the laws of distribution, Londres, Macmillan.

Young (Allyn A.), 1928, «Increasing returns and economic progress », Economic Journal, vol. XXXVIII, p. 527-542. 